\title{
A PARAMETER STUDY OF THE DUST AND GAS TEMPERATURE IN A FIELD OF YOUNG STARS
}

\author{
Andrea Urban ${ }^{1}$, Neal J. Evans II ${ }^{1}$, and Steven D. Doty ${ }^{2}$ \\ ${ }^{1}$ Department of Astronomy, University of Texas, Austin, TX 78712, USA; aurban@astro.as.utexas.edu, nje@astro.as.utexas.edu \\ ${ }^{2}$ Department of Physics and Astronomy, Denison University, Granville, OH 43023, USA; doty@ denison.edu \\ Received 2007 October 8; accepted 2009 April 7; published 2009 May 29
}

\begin{abstract}
We model the thermal effect of young stars on their surrounding environment in order to understand clustered star formation. We take radiative heating of dust, dust-gas collisional heating, cosmic-ray heating, and molecular cooling into account. Using DUSTY, a spherical continuum radiative transfer code, we model the dust temperature distribution around young stellar objects with various luminosities and surrounding gas and dust density distributions. We have created a grid of dust temperature models, based on our modeling with DUSTY, which we can use to calculate the dust temperature in a field of stars with various parameters. We then determine the gas temperature assuming energy balance. Our models can be used to make large-scale simulations of clustered star formation more realistic.
\end{abstract}

Key words: ISM: structure - methods: analytical - methods: numerical - radiative transfer - stars: formation

Online-only material: color figure

\section{INTRODUCTION}

Most of the stars in our Galaxy form in groups or clusters (Lada \& Lada 2003). Therefore, in order to understand the star formation history, the shape of the mass function, and the formation of massive $\left(M \gtrsim 5 M_{\odot}\right)$ stars in our Galaxy, the star formation process must be studied in its most common environment - a cluster. As stars form from their initial reservoir of gas and dust, they interact with their environment and heat the surrounding material, thus affecting future star formation. One of the first effects a protostar has on its environment is radiative heating from the accretion luminosity, gravitational contraction, and, subsequently, nuclear fusion. The radiation efficiently heats the dust, which in turn heats the gas through collisions. Young stars also affect their environment via strong winds and ionization, but ionizing photons are significant only when stars have grown to significant masses. We seek to isolate the separate effects by considering the effects of the star heating the gas via the dust.

Several groups use large-scale computer simulations to model clustered star formation. This is a complicated process requiring many assumptions in order to make the problem tractable. Klessen et al. (1998) and Martel et al. (2006) assume that the gas is isothermal. Bate et al. (2003) go beyond this assumption by using a barotropic equation of state. However, until recently, no one has included the effect of radiatively heating the dust and gas by the stars formed in the simulation. Krumholz et al. (2007) have included an approximate radiative transfer method, which works well in optically thick regions. Their method assumes that the gas temperature is equal to the dust temperature throughout their simulation. This approximation is only valid at high densities when the dust and gas are collisionally coupled. The method we develop explores the density space between isothermal and optically thick conditions. In this regime, we probe the volume that is far enough from stars so that dust grains have not been destroyed by the high temperatures close to stars, yet is not so far that we are in the regime where the external interstellar radiation field dominates heating.

In our method we include various heating and cooling processes to calculate the dust and gas temperature fields around young stars. Stars can heat dust grains more effectively than the gas because dust grains have broadband absorption properties.
Although we will not be explicitly modeling the motion or energy density of dust grains, we assume the dust and gas are well mixed and the dust grains transfer energy to gas particles through collisions using the energy transfer rate discussed in Young et al. (2004). The gas is heated by collisions with hot dust grains and cosmic rays. It can cool through carbon monoxide (CO) and other molecular line emission. Our method does not include direct gas heating from short wavelength stellar photons (i.e., UV and higher) which will only affect regions very close ( $\lesssim 100 \mathrm{AU})$ to stellar sources or near the cloud edge, where the interstellar radiation field is important. We consider a dense region lying within a region of fairly high extinction due to the parent molecular cloud.

In this paper, we use calculations of the dust and gas temperature fields around individual stars to calculate the dust and gas temperature fields in a field of stars. The dust temperature around a single source is calculated using a lookup table which we develop here. With this look-up table and an approximation to the flux-temperature conversion, we can calculate the dust temperature in a field of stars. Our lookup table is needed since the calculation of a single dust temperature distribution can take longer than a minute on current desktops and would take a substantial fraction of a large-scale simulation's computations. Therefore, we outline a method that can be used to decrease the time spent on the calculation of the dust temperature in future studies of clustered star formation.

With the calculated value of the dust temperature, we derive the gas temperature for a distribution of stellar sources, as in a young stellar cluster. The effect that protostars have on heating their environment using a hydrodynamic and gravity simulation will be addressed in a future paper.

In this paper, we first discuss the calculation of the dust temperature for single and multiple sources (Section 2), then we describe our gas temperature calculation (Section 3), and finally, we show some dust and gas temperature distributions in a field of sources (Section 4).

\section{DUST TEMPERATURE CALCULATION}

We consider two methods of calculating the dust temperature when there are multiple heating sources. The first approach 
(Analytic) assumes radiative equilibrium after summing up the flux of multiple sources heating a dust grain. This approach makes simplifying assumptions about the dust absorption and emission properties. The second approach (Numerical) uses the one-dimensional spherical radiative transfer code DUSTY (Nenkova et al. 2000) to calculate the dust temperature distribution around individual objects which have a variety of properties. Using the results of DUSTY, we convert the temperatures around individual objects to energy densities, which are then summed together over all of the objects in the field, and finally converted back to dust temperatures (assuming radiative equilibrium). In the following sections, we discuss the Analytic approach (Section 2.1) and the Numerical approach (Section 2.2) of calculating the dust temperature. Then we compare and analyze the two approaches (Section 2.3).

\subsection{Analytic Dust Temperature Calculation}

In order to analytically calculate the temperature of a dust grain in a field of $N$ stars we assume radiative equilibrium between the energy emitted by the heated dust grain and the energy absorbed by the dust grain from the radiation field of the surrounding stars. We calculate the dust temperature analytically using two methods and then compare them.

The rate of energy emitted by a single dust grain with radius $a$ is

$$
\left(\frac{d E}{d t}\right)_{\mathrm{em}}=4 \pi \int_{0}^{\infty} B_{v}\left(T_{d}\right) \sigma_{\nu} d \nu
$$

where $\sigma_{v}$ is the effective cross section of the grain as a function of frequency, $B_{v}\left(T_{d}\right)$ is the Planck function, and $T_{d}$ is the temperature of the dust grain. The rate of energy absorbed by the dust grain in a field of $N$ stars is

$$
\left(\frac{d E}{d t}\right)_{\mathrm{abs}}=\sum_{i=1}^{N} \frac{R_{* i}^{2}}{\left(\Delta \mathbf{r}_{* i}\right)^{2}} \int_{0}^{\infty} S_{\nu i} \sigma_{\nu} d \nu
$$

where $S_{v i}$ is the flux density at the stellar surface of star $i$ (which we assume is a blackbody at the stellar temperature, i.e., $\left.S_{v i}=\pi B_{v}\left(T_{* i}\right)\right), R_{* i}$ is the radius of star $i$, and the separation between star $i$ and the dust grain is $\Delta \mathbf{r}_{* i}=\left|\mathbf{r}_{* i}-\mathbf{r}\right|$, where $\mathbf{r}_{* i}$ is the position of star $i$ and $\mathbf{r}$ is the position of the dust grain.

Using $L_{* i}=4 \pi R_{* i}^{2} \sigma_{\mathrm{SB}} T_{* i}^{4}\left(L_{* i}\right.$ is the luminosity of star $i$ and $\sigma_{\mathrm{SB}}$ is the Stefan-Boltzmann constant) and assuming radiative equilibrium, Equations (1) and (2) combine to give

$$
\begin{aligned}
& \frac{\pi}{4 \pi \sigma_{\mathrm{SB}}} \sum_{i=1}^{N} \frac{L_{* i}}{T_{* i}^{4}\left(\Delta \mathbf{r}_{* i}\right)^{2}} \int_{0}^{\infty} B_{\nu}\left(T_{* i}\right) \sigma_{\nu} d v \\
& \quad=4 \pi \int_{0}^{\infty} B_{\nu}\left(T_{d}\right) \sigma_{\nu} d \nu .
\end{aligned}
$$

The effective cross section of the grain, $\sigma_{\nu}$, can be written in terms of the physical cross section of the grain, $\pi a^{2}$, and an efficiency factor, $Q_{\nu}$ that describes how well the grain absorbs or emits light at a particular frequency: $Q(\nu)=\sigma_{\nu} / \pi a^{2}$.

Then, assuming

$$
Q(v)=Q\left(v_{o}\right)\left(\frac{v}{v_{o}}\right)^{\beta},
$$

(where $v_{o}$ is a fiducial frequency) Equation (3) becomes

$$
\begin{aligned}
& \frac{1}{16 \pi \sigma_{\mathrm{SB}}} \sum_{i=1}^{N} \frac{L_{* i}}{T_{* i}^{4}\left(\Delta \mathbf{r}_{* i}\right)^{2}} \int_{0}^{\infty} B_{v}\left(T_{* i}\right) Q\left(v_{o}\right)\left(\frac{v}{v_{o}}\right)^{\beta} d v \\
& =\int_{0}^{\infty} B_{v}\left(T_{d}\right) Q\left(v_{o}\right)\left(\frac{v}{v_{o}}\right)^{\beta} d \nu .
\end{aligned}
$$

In the following subsections, we show an approximation for $T_{d}$ that is often made for hot stars and then provide a simple improvement that allows an analytic solution for cool stars as well. The purpose is to provide convenient points of comparison for the more correct calculations using radiative transport, described later in Section 2.2.

\subsubsection{Hot Stars}

Hot stars emit most of their luminosity in the UV and if we assume that grains absorb like graybodies in the $\mathrm{UV}$, then $\beta=0$ and $Q(v)=Q(\mathrm{UV})$ for the left-hand side of Equation (5). ( $Q(\mathrm{UV})$ is the flux weighted average absorption efficiency in the UV, which is discussed in greater detail later in this section.) Equation (5) then becomes

$$
\begin{gathered}
\frac{1}{16 \pi \sigma_{\mathrm{SB}}} \frac{Q(\mathrm{UV})}{\left(v_{\mathrm{UV}}\right)^{0}} \frac{2 h}{c^{2}} \sum_{i=1}^{N} \frac{L_{* i}}{T_{* i}^{4}\left(\Delta \mathbf{r}_{* i}\right)^{2}}\left(\frac{k T_{* i}}{h}\right)^{4+0} I_{4+0} \\
=\frac{Q\left(v_{o}\right)}{v_{o}^{\beta}} \frac{2 h}{c^{2}}\left(\frac{k T_{d}^{4}}{h}\right)^{4+\beta} I_{4+\beta},
\end{gathered}
$$

where

$$
I_{4+\beta}=\Gamma(4+\beta) \zeta(4+\beta)
$$

and the functions $\Gamma(x)$ and $\zeta(x)$ are defined as the gamma and Riemann zeta functions. Then solving Equation (6) for $T_{d}$ gives

$$
\begin{aligned}
T_{d}(\mathbf{r})= & {\left[\frac{3.89 \times 10^{4} \mathrm{~K}^{4}}{I_{4+\beta}} \frac{Q(\mathrm{UV})}{Q\left(v_{o}\right)}\left(\frac{h v_{o}}{k}\right)^{\beta}\right.} \\
& \left.\times \sum_{i=1}^{N} \frac{L_{* i} / 1 L_{\odot}}{\left(\Delta \mathbf{r}_{* i} / 1000 \mathrm{AU}\right)^{2}}\right]^{1 /(4+\beta)} .
\end{aligned}
$$

Note that the dependence on stellar temperature cancels out under these assumptions. From Equation (8), the form of the dust temperature profile can be written as

$$
T_{d}=K(\beta)\left[\sum_{i=1}^{N} \frac{L_{* i} / 1 L_{\odot}}{\left(\Delta \mathbf{r}_{* i} / 1000 \mathrm{AU}\right)^{2}}\right]^{1 /(4+\beta)},
$$

where $K$ (in units of Kelvin) and $\beta$ (dimensionless) are determined by the dust properties.

The value of $K$ is determined by $Q(\mathrm{UV}) / Q(125 \mu \mathrm{m})$ and $\beta$. Values of $Q(\mathrm{UV}) / Q(125 \mu \mathrm{m})$ from the literature are given in Table 1 . We can also calculate $Q(\mathrm{UV}) / Q(125 \mu \mathrm{m})$ from an adopted dust model. Our adopted dust model, "OH5 dust," is a combination of dust from Ossenkopf \& Henning (1994) and Pollack et al. (1994) as described in Young \& Evans (2005; see Figure 1). For OH5 dust, we calculate the value of $Q(\mathrm{UV}) / Q(125 \mu \mathrm{m})$ assuming a $10,000 \mathrm{~K}$ blackbody. $Q(\mathrm{UV})$ is calculated as the stellar flux weighted average absorption efficiency of OH5 dust. Therefore,

$$
\frac{Q(\mathrm{UV})}{Q(125 \mu \mathrm{m})}=\left(\frac{\sum_{\lambda=0.15 \mu \mathrm{m}}^{0.3 \mu \mathrm{m}} F_{\lambda} \sigma(\lambda)}{\sum_{\lambda=0.15 \mu \mathrm{m}}^{0.3 \mu \mathrm{m}} F_{\lambda}}\right) / \sigma(125 \mu \mathrm{m})=253,
$$




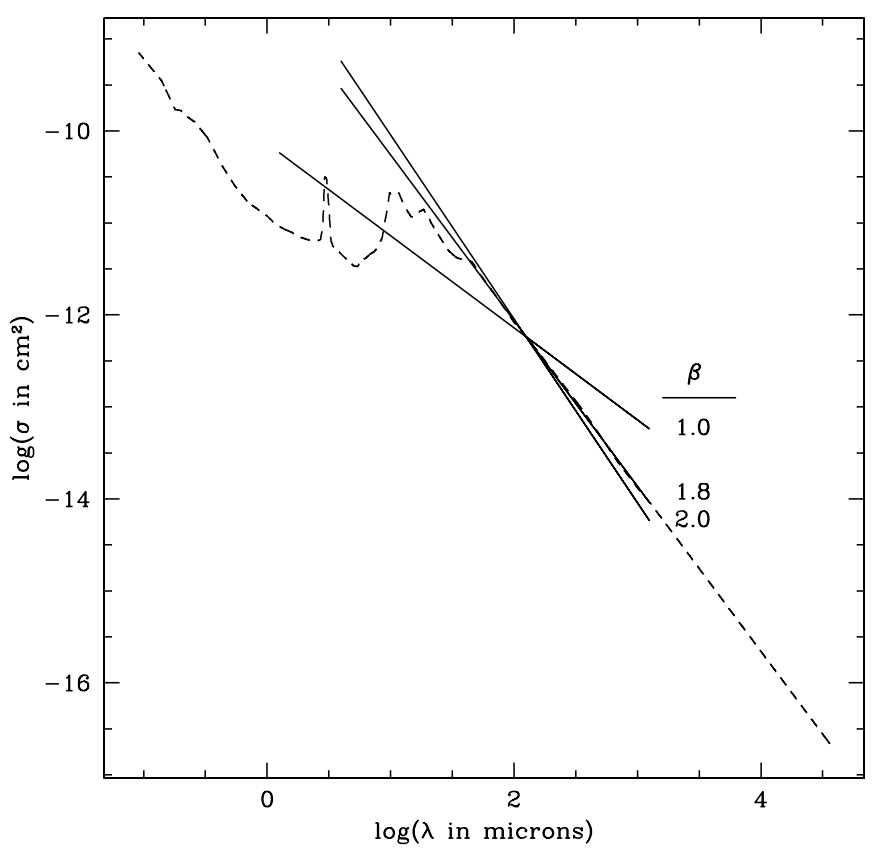

Figure 1. OH5 dust properties. Dashed line shows the variation of cross section with wavelength for OH5 dust. The solid lines show the different values of $\beta$ normalized at $125 \mu \mathrm{m}$ that we consider in Table 1.

Table 1

Dust Parameters for Sections 2.1.1 and 2.1.2

\begin{tabular}{lcccc}
\hline \hline Dust: Section 2.1.1 & $\frac{Q(\mathrm{UV})}{Q(125 \mu \mathrm{m})}$ & $K(1)$ & $K(1.8)$ & $K(2)$ \\
\hline Hildebrand (1983) & 4000 & $59.1 \mathrm{~K}$ & $52.4 \mathrm{~K}$ & $37.6 \mathrm{~K}$ \\
Makinen et al. (1985) & 790 & $42.7 \mathrm{~K}$ & $\ldots$ & $\ldots$ \\
OH5 (our dust model) & 253 & $34.0 \mathrm{~K}$ & $32.5 \mathrm{~K}$ & $23.7 \mathrm{~K}$ \\
\hline Dust: Section 2.1.2 & $T_{* i}$ & $K$ & $\beta$ & \\
\hline OH5 (our dust model) & $5000 \mathrm{~K}$ & $23.2 \mathrm{~K}$ & 1.66 & \\
OH5 (our dust model) & $10,000 \mathrm{~K}$ & $28.7 \mathrm{~K}$ & 1.66 & \\
\hline
\end{tabular}

where we take the range from 0.15 to $0.3 \mu \mathrm{m}$ as a rough representation of the UV range, which is relevant for a $10,000 \mathrm{~K}$ blackbody.

In Figure 1, we compare $\mathrm{OH} 5$ dust to an analytic approximation of $Q(v)$ (see Equation (4)) normalized at $125 \mu \mathrm{m}$ and vary the value of $\beta$, the dust grain's efficiency exponent. The line with $\beta=1.8$ fits well at long wavelengths but not at shorter wavelengths. The opposite is true for $\beta=1.0$. In Table 1 , we have listed all of the values of $Q(\mathrm{UV}) / Q(125 \mu \mathrm{m})$ that we consider. Based on the analysis of Figure 1, we vary the value of $\beta$ to $1,1.8$, and 2 . Then we calculate the values of $K$ corresponding to the different values of $\beta$ (except in the case of Makinen et al. 1985 where $\beta=1$ by definition).

\subsubsection{Cool Stars}

We also consider cooler stars with $T_{*}=5000 \mathrm{~K}$. For stars at this temperature, most of the stellar light is not coming from the UV, in contrast to the hotter stars discussed in the previous section. To calculate the dust temperature in a field of cool stars we start from Equation (3). We use the information in Figure 1 to derive the dust temperature profile in the form of Equation (9) by solving the integrals in Equation (3) numerically. We can then derive values of $K$ and $\beta$ for Equation (9). Since this method makes no assumptions about the shape of the input spectra, we

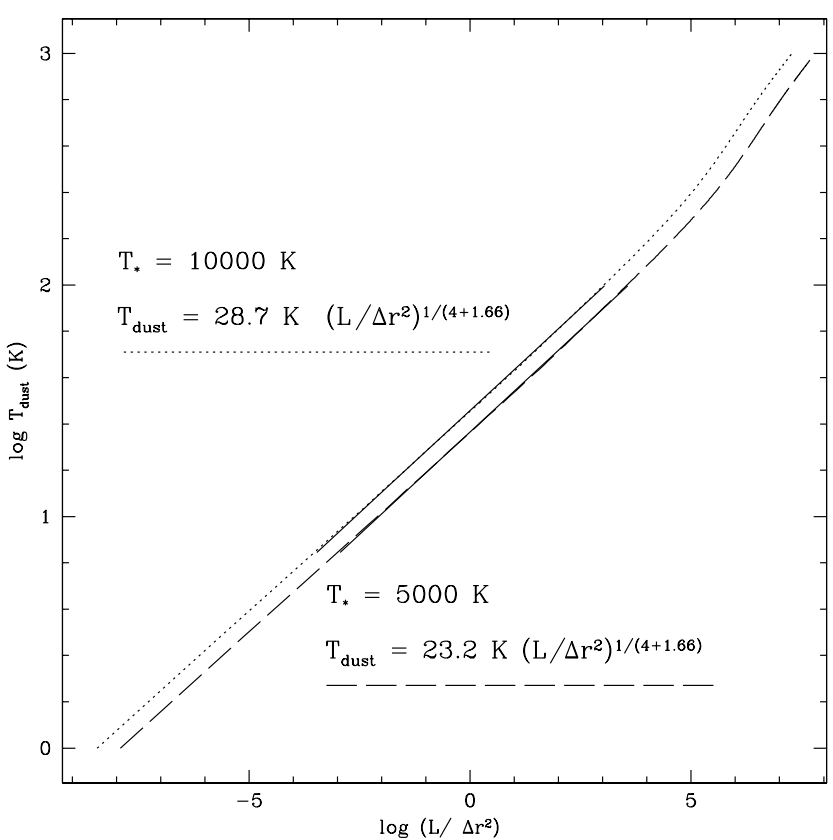

Figure 2. Relationship of $T_{d}$ and $L / \Delta r^{2}$ assuming a stellar temperature of $T_{*}=5000 \mathrm{~K}$ (dashed) or $T_{*}=10,000 \mathrm{~K}$ (dotted). The dashed and dotted lines show the value derived from solving Equation (3). The solid lines overplotted on the dashed and dotted lines are the least-squares best fit to the respective line between $T_{d}=5$ and $100 \mathrm{~K}$. Fit parameters are given in the figure. For $T_{*}=5000 \mathrm{~K}$ (dashed), $K=23.2 \mathrm{~K}$ and $\beta=1.66$. For $T_{*}=10,000 \mathrm{~K}$ (dashed), $K=28.7 \mathrm{~K}$ and $\beta=1.66$. The units of $L, \Delta r$, and $T_{d}$ are $1 L_{\odot}$, $1000 \mathrm{AU}$, and $1 \mathrm{~K}$, respectively. For reference, for $1 L_{\odot}, 10,000 \mathrm{~K}$ blackbody at a distance of $1000 \mathrm{AU}$, a dust grain's temperature is $23.2 \mathrm{~K}$. For a star of $10^{3} L_{\odot}$ with the same temperature at the same distance, the dust grain's temperature is approximately $80 \mathrm{~K}$.

calculate $K$ and $\beta$ for $T_{*}=5000 \mathrm{~K}$ and $10,000 \mathrm{~K}$ and list them in Table 1. In Figure 2, we show the behavior of $T_{d}$ with $L / \Delta r^{2}$. We only fit the temperature regime between $5 \mathrm{~K}$ and $100 \mathrm{~K}$ since the behavior at higher temperatures is not linear in $\log T-\log \left(L / \Delta r^{2}\right)$ space and dust temperatures below $5 \mathrm{~K}$ are unrealistic.

\subsubsection{Summary}

We have calculated an analytic solution to the dust temperature in a field of stars which depends only on the stellar temperature and the properties of the dust. We have discussed two methods of calculating the dust temperature. The first allows only hot stars and the second permits any stellar temperature. We do not find a large scatter in the values of $K$ and $\beta$ listed in Table 1 considering the different assumptions made about the dust properties. In particular, the agreement between the value of $K(1.8)=32.5 \mathrm{~K}$, which approximates the UV contribution, and $K=28.7 \mathrm{~K}$ for $T_{*}=5000 \mathrm{~K}$, which uses exact integration, is worth noting. We cannot address how a range of stellar temperatures would affect the dust temperature using the analytic method we describe, i.e., variable $T_{* i}$ in Equation (3). Another assumption we have made in this section is the optically thin approximation. In order to calculate the temperature of the dust we have assumed that all of the grains are isolated from each other and are only heated by the stellar radiation field. In reality, the presence of grains will alter the radiation field as the grains process and re-emit the incident radiation. This will alter the radiation field assumed in Equation (2). In order to compare the analytic solutions described in this section to the numerical 
calculation in Section 2.2, we define the "Analytic Solution" as Equation (9) with $K=23.2 \mathrm{~K}$ and $\beta=1.66\left(T_{* i}=5000 \mathrm{~K}\right)$ throughout the remainder of the paper.

\subsection{Numerical Dust Temperature Calculation}

Our more correct method of calculating the dust temperature uses DUSTY (Nenkova et al. 2000), a one-dimensional spherical radiative transfer code. In order to calculate the dust temperature in a field of stars, we use DUSTY to first calculate the dust temperature around each star in the field as if it were isolated. Then we use these results to calculate the temperature in the field by assuming radiative equilibrium. Although it is more accurate to use DUSTY to calculate the dust temperature around a young star than the method described in Section 2.1, DUSTY can take over 1 minute to run for low optical depths-with yet longer run times for larger values of $\tau_{100 \mu \mathrm{m}}$. Therefore, we calculate the dust temperature profile for various combinations of luminosity, outer radius, and density profile to create a look-up table. (The parameters we consider are discussed in Section 2.2.1.) We use OH5 dust opacities as described in Young \& Evans (2005).

We approximate the dust temperature profile around a single source, $i$, with the expression

$$
T_{d}\left(\Delta \mathbf{r}_{* i}\right)=K_{i}\left(\frac{L_{* i}}{\left(\Delta \mathbf{r}_{* i}\right)^{2}}\right)^{1 /\left(4+\beta_{i}\right)}
$$

where $L$ is the input luminosity in units of $L_{\odot}, \Delta \mathbf{r}_{* i}$ is the distance from the star in units of $1000 \mathrm{AU}$, and $K$ and $\beta$ are functions of the density profile and dust properties. (The units of $L$ and $\Delta \mathbf{r}_{* i}$ will remain $L_{\odot}$ and $1000 \mathrm{AU}$ throughout the remainder of the paper.) This simple profile is a valid assumption when the dust is optically thin. Although the gas and dust are denser closer to the central source and likely to be optically thick there, we are mainly interested in the dust temperature distribution far from the central source where the physical processes we consider are dominant and the dust and gas are transparent to the cloud exterior for long wavelength emission.

For each set of parameters, we run DUSTY and solve for a value of $K$ and $\beta$ (see Section 2.2.1). Since we are only interested in the dust temperature far from the source, we fit the outer $25 \%$ of the dust temperature profile obtained from DUSTY in $\log T-\log r$ space using least-squares fitting in order to determine the values of $\beta$ and $K$ in Equation (11). We call these values of $\beta$ and $K$ our "Fit Solution."

In order to calculate the dust temperature of a region heated by more than one protostar, we add up the flux at the region of interest using

$$
F(\mathbf{r})=\sum_{i=1}^{N} \frac{L_{* i}}{4 \pi\left(\Delta \mathbf{r}_{* i}\right)^{2}} .
$$

Combining Equations (11) and (12), we derive

$$
F(\mathbf{r})=\sum_{i=1}^{N} \frac{\left(T_{d}\left(\Delta \mathbf{r}_{* i}\right) / K_{i}\right)^{4+\beta_{i}}}{4 \pi} .
$$

In order to be able to convert to temperature later, we approximate Equation (13) by using averages and we assume

$$
F(\mathbf{r})=\frac{\left(T_{d}(\mathbf{r}) / \bar{K}\right)^{4+\bar{\beta}}}{4 \pi}
$$

where $\bar{K}$ and $\bar{\beta}$ are defined as the flux-weighted averages of the $K$ and $\beta$ values that contribute to the flux at point $\mathbf{r}$, i.e.,

$$
\bar{\beta}=\frac{\sum \beta_{i} L_{* i} / 4 \pi \Delta \mathbf{r}_{* i}^{2}}{\sum L_{* i} / 4 \pi \Delta \mathbf{r}_{* i}^{2}}
$$

and

$$
\bar{K}=\frac{\sum K_{i} L_{* i} / 4 \pi \Delta \mathbf{r}_{* i}^{2}}{\sum L_{* i} / 4 \pi \Delta \mathbf{r}_{* i}^{2}},
$$

where the sums are from $i=1$ to the total number of stars, $N$.

Therefore, equating Equations (13) and (14) and solving for $T_{d}(\mathbf{r})$ gives

$$
T_{d}(\mathbf{r})=\bar{K}\left[\sum_{i=1}^{N}\left(\frac{T\left(\Delta \mathbf{r}_{* i}\right)}{K_{i}}\right)^{4+\beta_{i}}\right]^{1 /(4+\bar{\beta})} .
$$

Using Equation (11), we obtain

$$
T_{d}(\mathbf{r})=\bar{K}\left[\sum_{i=1}^{N} \frac{L_{* i}}{\Delta \mathbf{r}_{* i}^{2}}\right]^{1 /(4+\bar{\beta})},
$$

which is the equation we use to calculate the dust temperature in a field of sources.

Equation (18) is of course an approximation; full threedimensional radiative transport calculations are needed to produce a complete description, but this approximation captures the essence of multiple sources. In Figure 3, we compare the dust temperature calculated using our DUSTY Fit Solutions around two single sources to the dust temperature calculated using Equation (18) between the same two sources separated by some distance. The distance is calculated by specifying $n_{\mathrm{eq}}$, the density at which the envelope density profiles of the two individual sources are equal. We compare two models (source 1: $K=$ $19.5 \mathrm{~K}, \beta=1.78$ and source $2: K=22.9 \mathrm{~K}, \beta=1.78$; see Figure 3 for more details) that represent the average values in our parameter space. We also compare an average model (source 1: $K=20.6 \mathrm{~K}, \beta=1.72$ ) to a model that has a low value of $\beta$ (source 2 : $K=18.7 \mathrm{~K}, \beta=1.17$ ), but keep the luminosity the same between the two $\left(L=10^{2} L_{\odot}\right)$.

As expected, we find that the dust temperature at some distance from the individual sources is greater using Equation (18) ("Flux Weighted" in Figure 3) which attempts to add the individual fluxes than the temperature calculated around the individual sources ("DUSTY-fit" in Figure 3). However, this only occurs in a very small region between the two sources and the temperature is underestimated by $\lesssim 30 \%$ in the majority of the space between the sources. The largest difference between the two temperatures is in the space very close to the sources. But, as we discuss later in Section 2.3, we expect to incorrectly model the temperature close to the sources using our method of deriving $K$ and $\beta$.

\subsubsection{Parameter Space}

We assume the dust and gas are well mixed and have the same density profile, offset only by the dust-to-gas mass ratio $\left(\eta_{\mathrm{dg}}\right)$ described in Section 3, i.e., $\rho_{\text {dust }}=\eta_{\mathrm{dg}} \rho_{\text {gas }}$. We assume $N_{\mathrm{H}_{2}} / N_{\mathrm{He}}=5$, which gives $\mu=2.33$. The density profile of the gas is parameterized with $n_{\mathrm{o}}$ and $\alpha$ using

$$
n_{\mathrm{gas}}=n_{o}\left(\frac{r}{1000 \mathrm{AU}}\right)^{-\alpha} \text {. }
$$




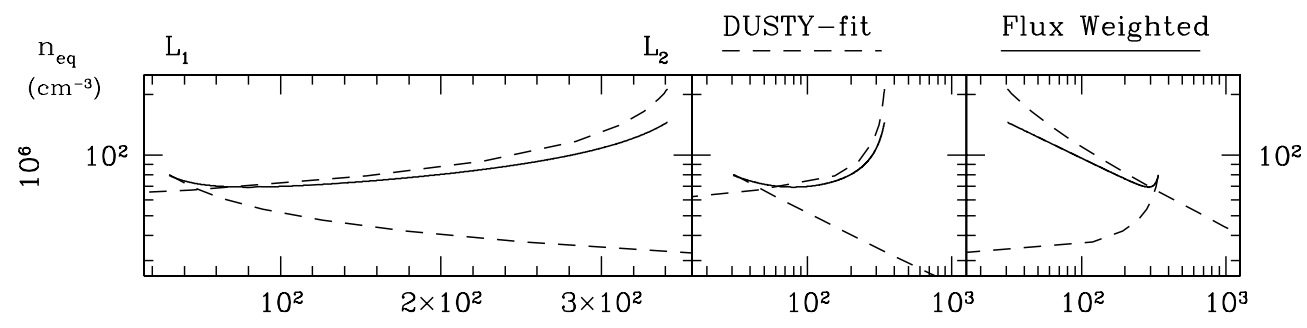

1: $\mathrm{L}=1 \mathrm{~L}_{\odot}, \mathrm{n}_{\mathrm{o}}=10^{3} \mathrm{~cm}^{-3}, \alpha=2.0, \mathrm{~K}=19.5 \mathrm{~K}, \beta=1.78 \quad 2: \mathrm{L}=10^{2} \mathrm{~L}_{\odot}, \mathrm{n}_{\mathrm{o}}=10^{5} \mathrm{~cm}^{-3}, \alpha=2.0, \mathrm{~K}=22.9 \mathrm{~K}, \beta=1.78$
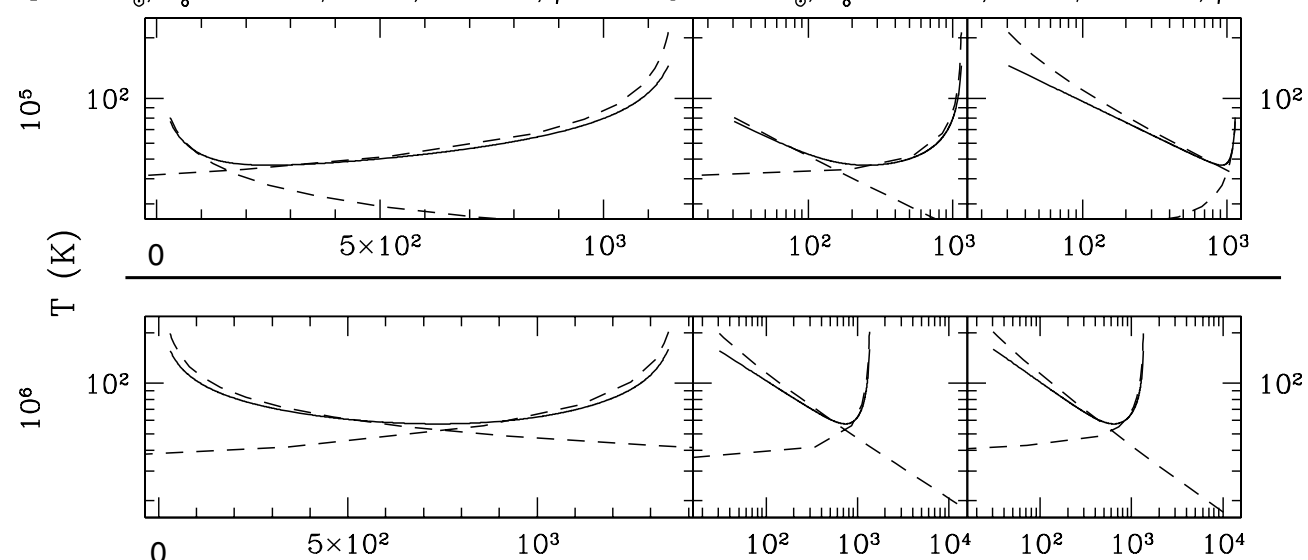

1: $\mathrm{L}=10^{2} \mathrm{~L}_{\odot}, \mathrm{n}_{\mathrm{o}}=10^{5} \mathrm{~cm}^{-3}, \alpha=1.5, \mathrm{~K}=20.6 \mathrm{~K}, \beta=1.722: \mathrm{L}=10^{2} \mathrm{~L}_{\odot}, \mathrm{n}_{\mathrm{o}}=10^{6} \mathrm{~cm}^{-3}, \alpha=1.0, \mathrm{~K}=18.7 \mathrm{~K}, \beta=1.17$

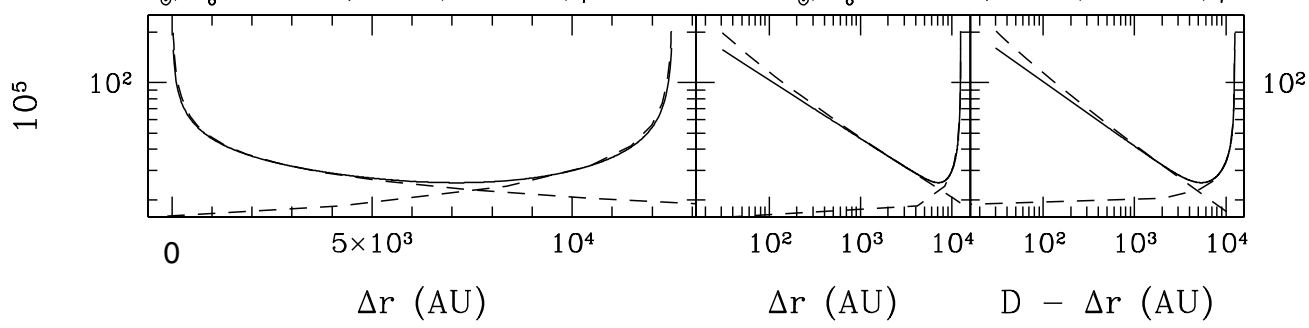

Figure 3. Comparison of individual DUSTY Fit Solutions (dashed line) to Flux Weighted Solution of Equation (18) (solid line). For the Flux Weighted Solution, the temperature between the two sources is calculated assuming a density distribution between them which meets at a density $n_{\mathrm{eq}}$ as quoted on the left-hand side of the plot. At a given value of $n_{\mathrm{eq}}$ we show three horizontal plots of the same data from different perspectives. On the longer plot on the left we use a linear scale from the perspective of source 1. The two smaller plots on the right use a logarithmic scale, from the perspective of source 1 (middle) and source 2 (right). This is done to show structure close to the individual sources. The model parameters we list in the figures are described in more detail in Section 2.2.1. For the top two plots, the source parameters are listed between them, similarly for the bottom two plots.

We model the entire parameter space listed in Table 2 with two exceptions. First, we limit the density at the inner radius to be less than $10^{10} \mathrm{~cm}^{-3}$, because at higher densities dynamical effects may become important since the free-fall time becomes small as the density increases. Second, we limit the mass of the envelope to be less than $\sim 1000 M_{\odot}$ since a larger envelope would likely produce a cluster of stars (assuming a star formation efficiency of $10 \%$ and a maximum stellar mass of $100 M_{\odot}$ ) which would break the assumption of spherical symmetry. Therefore, combinations of large/small $\alpha$ and large $n_{o}$ (for inner density limit/mass limit) may not be represented in our models. Based on these restrictions, of the 5049 possible models in our parameter space, we model 3231 or $64 \%$ of them. In Figure 4, we show the envelope masses modeled in our parameter space. Figure 5 shows the relationship between the values of $\tau_{100 \mu \mathrm{m}}$, $\alpha$, and $n_{o}$ for the models in our parameter space.

We chose the inner radius of the spherical dust-gas envelope to be fixed at $30 \mathrm{AU}$. We have varied the inner radius by a factor of 0.1 and 10 and the difference in the values of $K$ and $\beta$ is less than $1 \%$. Therefore, we conclude that the choice of the inner radius does not significantly affect our results. We also consider the possibility of dust destruction. The luminosity required to reach a dust destruction temperature of $1500 \mathrm{~K}$ at 30
Table 2

Dust Model Parameters

\begin{tabular}{lcccr}
\hline \hline Parameter & Lower Limit & Upper Limit & $\Delta^{\mathrm{a}}$ & $N^{\mathrm{b}}$ \\
\hline $\log \left(L / L_{\odot}\right)$ & -2 & 6 & 0.5 & 17 \\
$\log \left(n_{o} / \mathrm{cm}^{-3}\right)$ & 2.5 & 7.5 & 0.5 & 11 \\
$\alpha$ & 0 & 4 & 0.5 & 9 \\
$\log \left(r_{\text {out }} / 1 \mathrm{pc}\right)$ & -1 & 0 & 0.5 & 3 \\
\hline
\end{tabular}

Notes.

${ }^{\text {a }}$ Spacing of parameters.

${ }^{\mathrm{b}}$ Number of parameters.

AU using the Analytic Solution is $4.93 \times 10^{6} L_{\odot}$. This is outside our sample range of luminosities; therefore, we can neglect the effects of dust destruction. We also vary the outer radius, $r_{\text {out }}$, to obtain a range of values. We find that our values of $K$ and $\beta$ do not strongly depend on $r_{\text {out }}$.

In all cases, the stellar input spectrum is assumed to be a blackbody with $T_{*}=5000 \mathrm{~K}$. This value does not strongly influence the calculated temperature distribution at outer radii since the radiation is quickly reprocessed by the dust to longer wavelengths. Shirley et al. (2002) and van der Tak et al. (1999) show that varying the input spectrum has little effect on the 


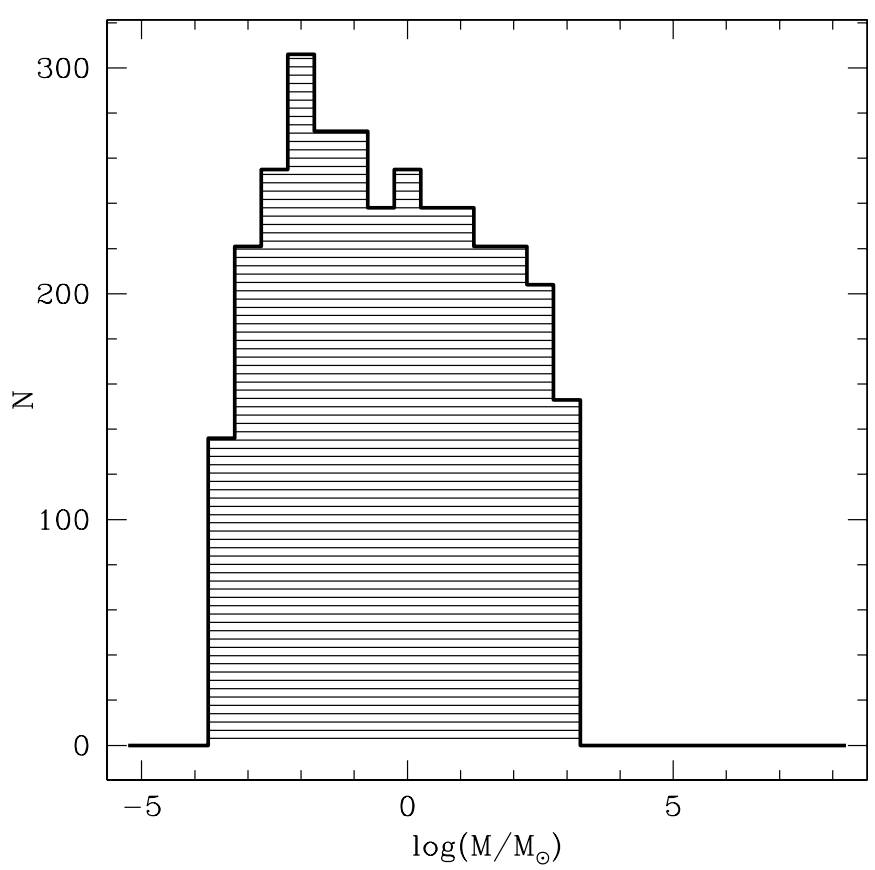

Figure 4. Histogram of envelope masses of models in our parameter space.

output spectrum. However, these authors have only modeled a handful of cases. Since we are covering a much larger range in parameter space, we investigate in greater detail the possible differences that may be caused by a higher blackbody temperature. We expect to see only differences in the extremes of our parameter space based on the work of the previous authors.

To test our prediction, we have modeled the entire parameter space discussed above using a blackbody with $T_{*}=10,000 \mathrm{~K}$. We compare the results in Figures 6 and 7. These figures show that the percentage difference between a model with a $10,000 \mathrm{~K}$ blackbody spectrum and the same model with a $5000 \mathrm{~K}$ blackbody is small (less than 10\%) in most of our parameter space. In Figure 6, we show that the models with the largest percentage differences are at the extremes of our parameter space at high density $\left(n_{o}>10^{5} \mathrm{~cm}^{-3}\right)$ and low $\alpha$. We find that among this group, the main parameter that causes a model to have a high percentage difference is the outer radius. Models with $r_{\text {out }} \leqslant 0.1 \mathrm{pc}$ have percentage differences greater than $50 \%$, as seen in Figure 7 . This can be explained by examining Figure 8 where an increase in density causes an increase in the sphere of influence of the nonlinear (in $\log T-$ $\log r$ space) region in the center of the model. Therefore, models with small outer radii and high densities will have different fractions of the nonlinear (or optically thick) region included in their calculation of the Fit Solution (recall that this is calculated using the outer $25 \%$ of the dust temperature profile in $\log T-$ $\log r$ space) compared to models with larger outer radii but the same density. Therefore, the largest difference in models with two different input temperatures occurs mainly for models with high densities, low $\alpha$ 's, and small outer radii.

\subsection{Comparison of Dust Temperature Calculation Methods}

Figures 8 and 9 compare the two methods of calculating the dust temperature around a single source. The Analytic Solution uses OH5 dust parameters as described in Section 2.1.3.

Figure 8 shows that the Analytic Solution captures the shape of the DUSTY temperature profile, but at high $\tau_{100 \mu \mathrm{m}}$ it

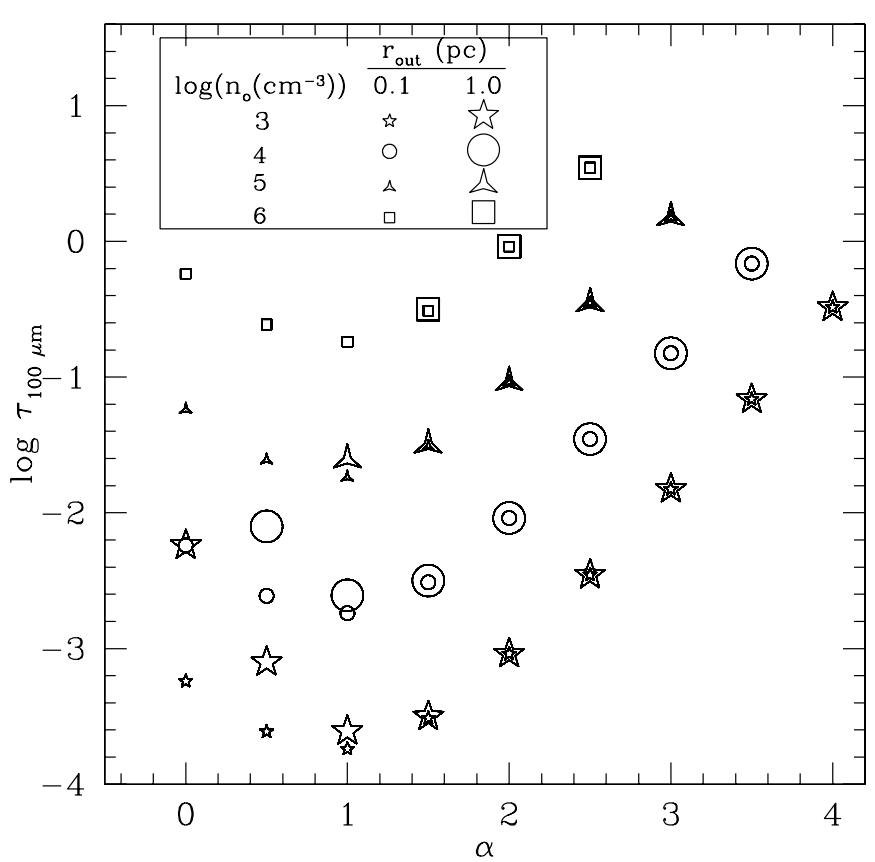

Figure 5. Relationship between $\alpha, \tau_{100 \mu \mathrm{m}}$, and $n_{o}$. This figure shows how $\tau_{100 \mu \mathrm{m}}$ varies with $\alpha, n_{o}$, and $r_{\text {out }}$ for our models. At a fixed value of $n_{o}$ and as $\alpha$ increases (and $\alpha>1$ ), $\tau_{100 \mu \mathrm{m}}$ increases as well due to the sharp density increase in the center of the density profile. For lower values of $\alpha(\alpha<1)$, $\tau_{100 \mu \mathrm{m}}$ begins to increase again, but this increase is due to the increase in the amount of material included in the profile at the edge, i.e., the value of $r_{\text {out }}$.

overestimates the magnitude. If we only vary the luminosity (see Figure 9), then all solutions (DUSTY, Analytic, and Fit) produce an increase in temperature, due to the increased luminosity. However, as seen in Figure 8, if we change other parameters, $n_{o}$, $\alpha$, and $r_{\text {out }}$, we cannot predict the effect on the Analytic Solution because it is independent of these parameters. The Fit Solution is a compromise between the Analytic and DUSTY Solutions. The Analytic Solution ignores the density profile and is unreliable when the optical depth is high. The DUSTY Solution takes too long to calculate. The Fit Solution, as described in Section 2.2, also provides the best fit at outer radii, compared to the analytic solution, as shown in Figures 8 and 9.

Figure 10 shows histograms of $K$ and $\beta$ derived from our Fit Solution for our parameter space. While most models cluster around the Analytic Solution, there is a spread that is dependent on some of the input parameters. In Figure 11, we show how the values of $K$ and $\beta$ depend on the $\alpha$ parameter. Models with low values of $\alpha$ tend to have $K$ and $\beta$ values that are far from the Analytic Solution (i.e., $K>30 \mathrm{~K}, \beta<1$ ).

In Figures 12 and 13, we show how different parameters determine the values of $K$ and $\beta$. Figure 12 shows that luminosity and the value of $K$ are positively correlated. Although we have explicitly removed the luminosity dependence from Equation (11), there is still some dependence of $K$ on luminosity. This can be understood in terms of radiative trapping. For high luminosities, more photons at shorter wavelengths exist farther from the star, which increases the size of the region of high optical depth and leads to an increase in the value of $K$. (This can also be seen in the values of $K$ listed in Figure 9). Also, as $\alpha$ increases, then $K$ decreases. Increasing $\alpha$ shrinks the size of the region of high optical depth due to the buildup of material close to the star. Figure 13 shows that as $\alpha$ decreases and luminosity increases, then $\beta$ decreases. The drastic drop of $\beta$ at high luminosities can be understood by comparing the two models shown in Figure 9. 

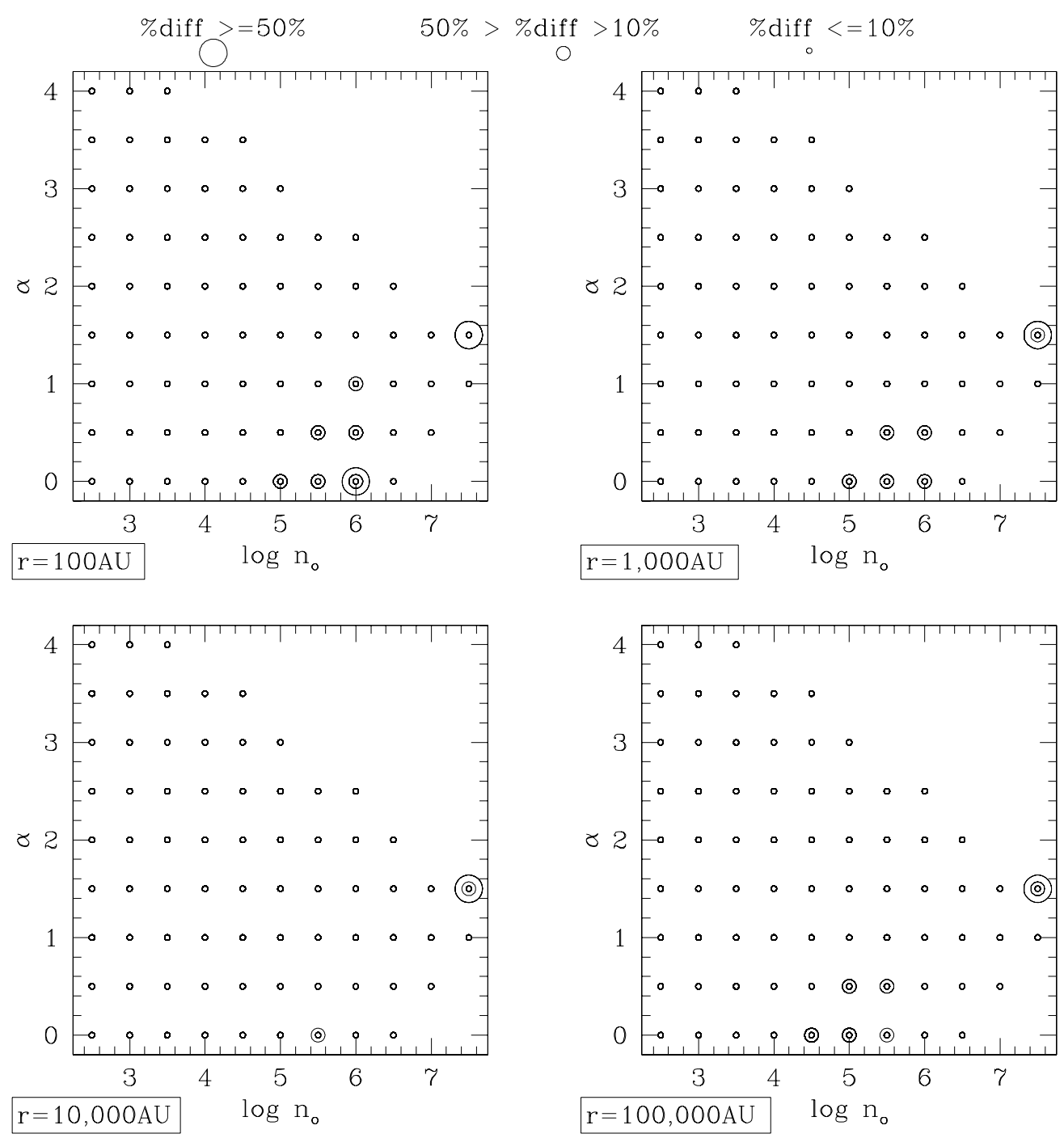

Figure 6. Dust temperature percent difference for $T_{*}=5000 \mathrm{~K}$ and $T_{*}=10,000 \mathrm{~K}$ models in $n_{o}\left(\mathrm{~cm}^{-3}\right)$ and $\alpha$ space. The distance that the dust temperature is sampled at is given in the box in the bottom left-hand corner of the individual figures and ranges from $100 \mathrm{AU}$ to 100,000 AU. The key to the percent difference is given at the top of the figure, with larger circles indicating a larger percent difference. Models at low $\alpha$ with high values of $n_{o}$ are not in our sample due to the maximum mass criterion. Models missing in the top right corner of the figures at high $n_{o}$ and high $\alpha$ are missing due to the criterion which sets the maximum density at the inner radius.

In the two models, the radius at which the dust temperature turns up (i.e., when the material changes from optically thin to optically thick) moves out in radius as the luminosity increases. Since we model the value of $\beta$ using only the outer $25 \%$ of the material, we expect to be considering only the optically thin material. However, as the luminosity increases, the radius at which the transition from optically thick to optically thin material moves outward. Therefore, at higher luminosities, our calculation of $\beta$ becomes influenced by the optically thick region. This is the reason that $\beta$ decreases and moves away from the optically thin Analytic Solution as luminosity increases.

In order to calculate values of $\beta$ and $K$ that are not modeled in our parameter space, we interpolate between known values in our look-up table. We use the method for interpolating in two or more dimensions described in chapter 3 of Press et al. (1992). This method involves solving successive one-dimensional interpolations. We modified the POLIN2 subroutine to interpolate in four dimensions. The actual method of interpolation that we used was the polynomial interpolation method over three known quantities from the subroutine POLINT in Press et al.
(1992). We tested our interpolation method by running extra models through DUSTY which were at intermediate points in our parameter space. Then we compared the results between the DUSTY Solution and solution from our interpolation method. For three of these intermediate models, we fix $n_{o}, \alpha$, and $r_{\text {out }}$ and vary the luminosity in order to explore how luminosity affects the interpolation method. We also explore the effect of varying $r_{\text {out }}$ and we study the behavior of the intermediate models near the edges of the $n_{o}-\alpha$ parameter space. We find that our interpolation method is quite accurate, with median differences of $0.55 \%$ and $0.09 \%$ for $K$ and $\beta$, respectively. The differences in $K$ and $\beta$ are all less than $2 \%$. The largest differences are for models with very high luminosities near $\sim 10^{6} L_{\odot}$. This is likely to be due to the rapid change of the value of $\beta$ with luminosity (see Figure 13).

Based on the previous discussion in this section, we use the Fit Solution described in Section 2.2 to calculate the dust temperature in the remainder of the paper. Since we are primarily interested in the dust temperature far from the luminosity source, we find that we can model the shape and magnitude of the dust 

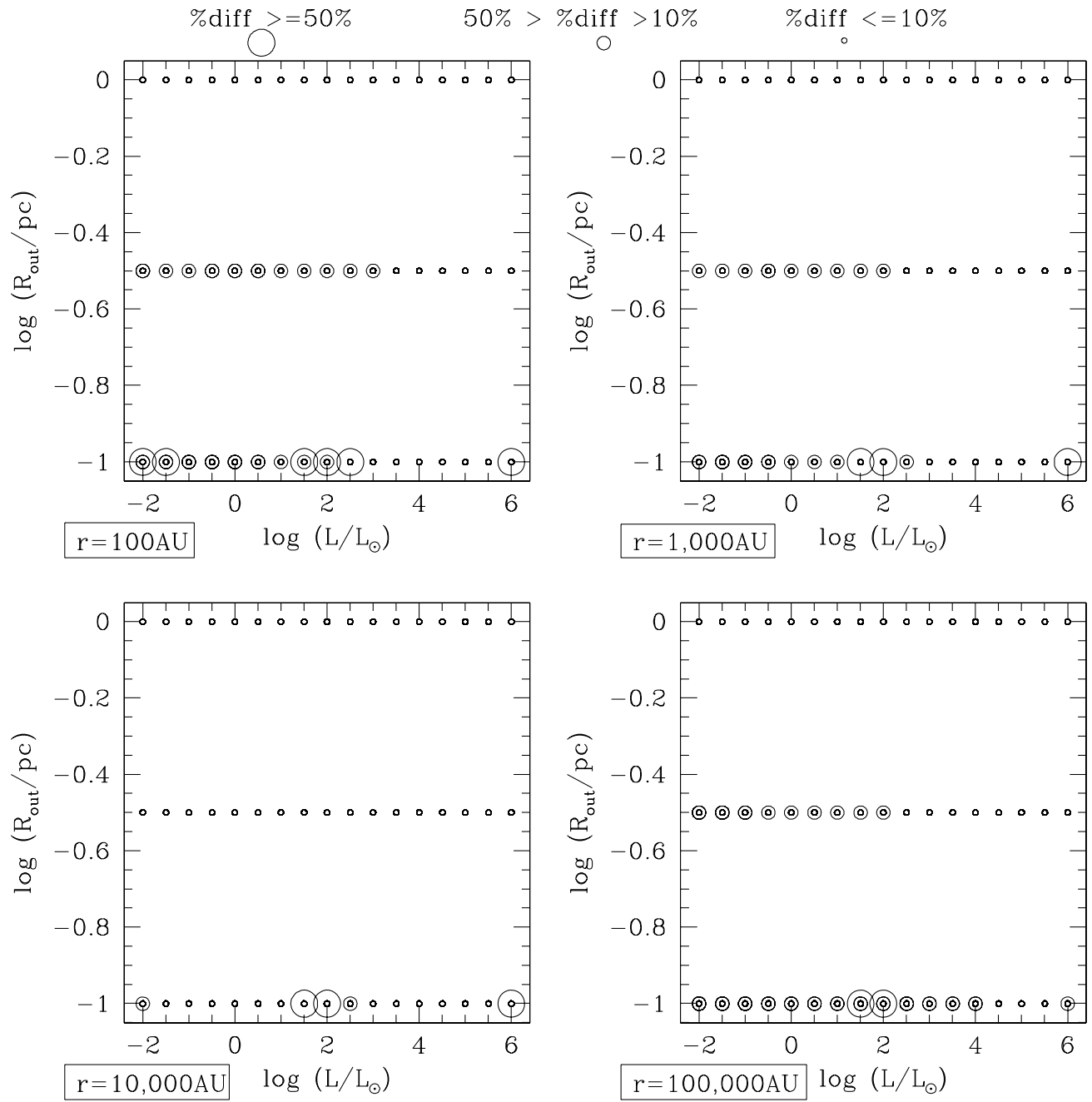

Figure 7. Dust temperature percent difference for $T_{*}=5000 \mathrm{~K}$ and $T_{*}=10,000 \mathrm{~K}$ models in $\log \left(L / L_{\odot}\right)$ and $\log \left(r_{\text {out }} / \mathrm{pc}\right)$ space. Similar to Figure 6 but for log $\left(L / L_{\odot}\right)$ and $\log \left(r_{\text {out }} / \mathrm{pc}\right)$.
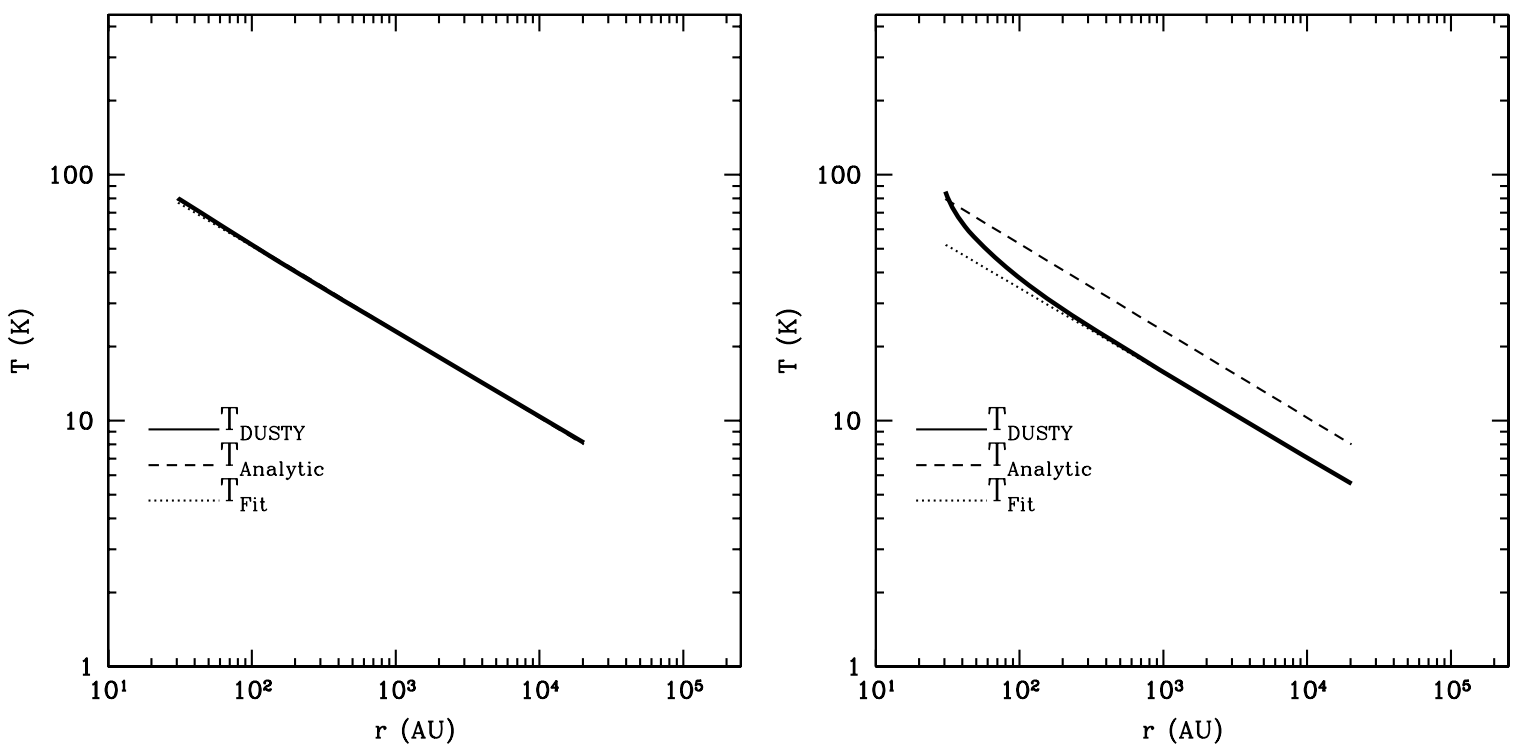

Figure 8. Comparison between temperature distributions for two different models with a low and high fiducial density. For both models, $L=1 L_{\odot}, \alpha=2$, and $r_{\text {out }}=0.1 \mathrm{pc}$. The figure on the left has $\log n_{o}=2.5$ and $\tau_{100 \mu \mathrm{m}}=2.894 \times 10^{-4}$. The fit parameters are $K=23.0 \mathrm{~K}$ and $\beta=1.78$. For this case, the Fit and Analytic Solutions lie nearly on top of the DUSTY Solution which makes them difficult to distinguish from the DUSTY Solution. The figure on the right has log $n_{o}=5.5$ and $\tau_{100 \mu \mathrm{m}}=2.894 \times 10^{-1}$ with $K=15.6 \mathrm{~K}$ and $\beta=1.81$. Although both of these models are optically thin (at most radii) as assumed in the Analytic Solution, it is clear that the Analytic Solution is not a good description of the dust temperature for both cases. 

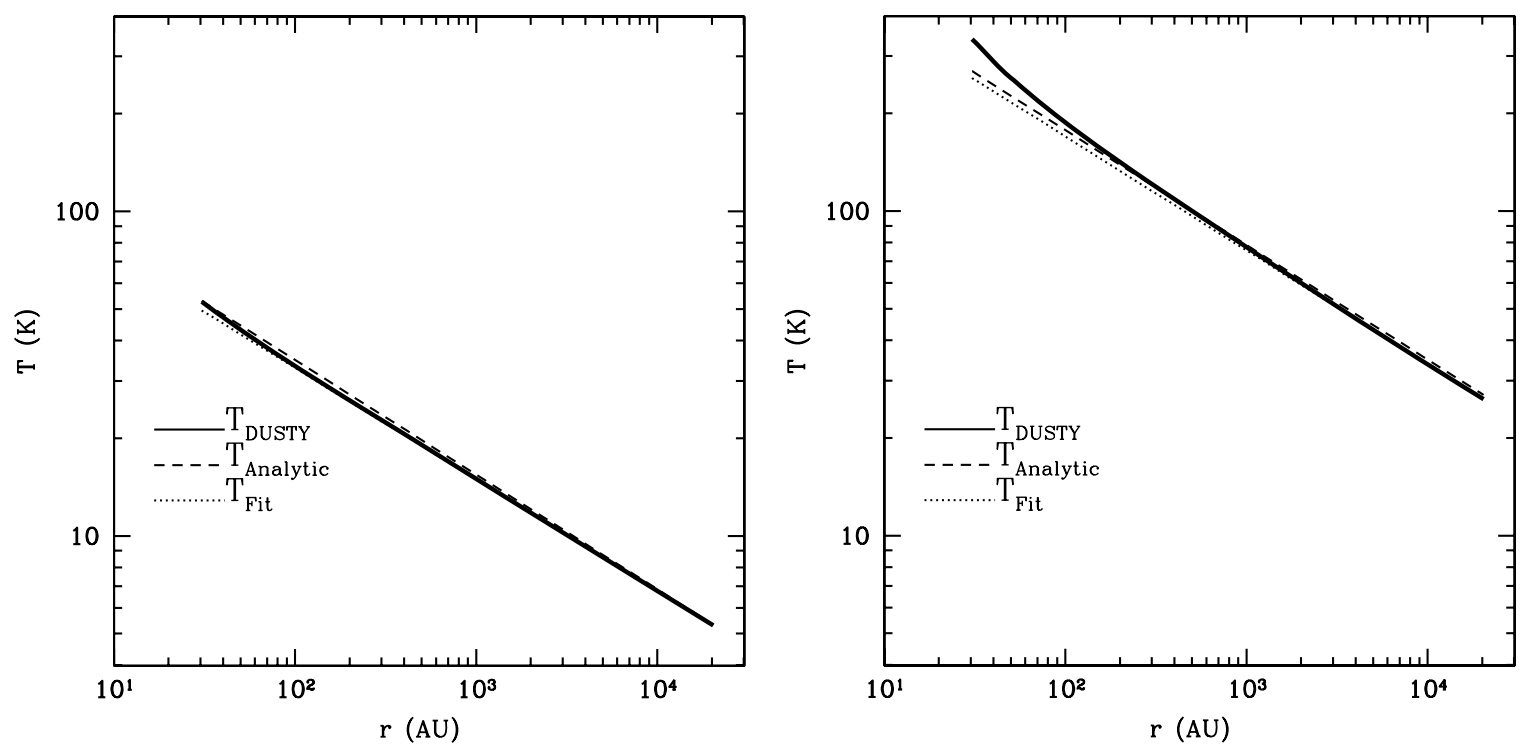

Figure 9. Comparison between temperature distributions for two different models with a low and high luminosity. For both models, $\alpha=3$, and $r_{\text {out }}=0.1$ pc, log $n_{o}=2.5$. The figure on the left has $\log L / L_{\odot}=-1$. The fit parameters are $K=22.2 \mathrm{~K}$ and $\beta=1.82$ The figure on the right has $\log L / L_{\odot}=3$ with $K=22.5 \mathrm{~K}$ and $\beta=1.71$. These models show how increasing the luminosity increases the overall dust temperature as well as making the optically thick region near the center extend farther out.
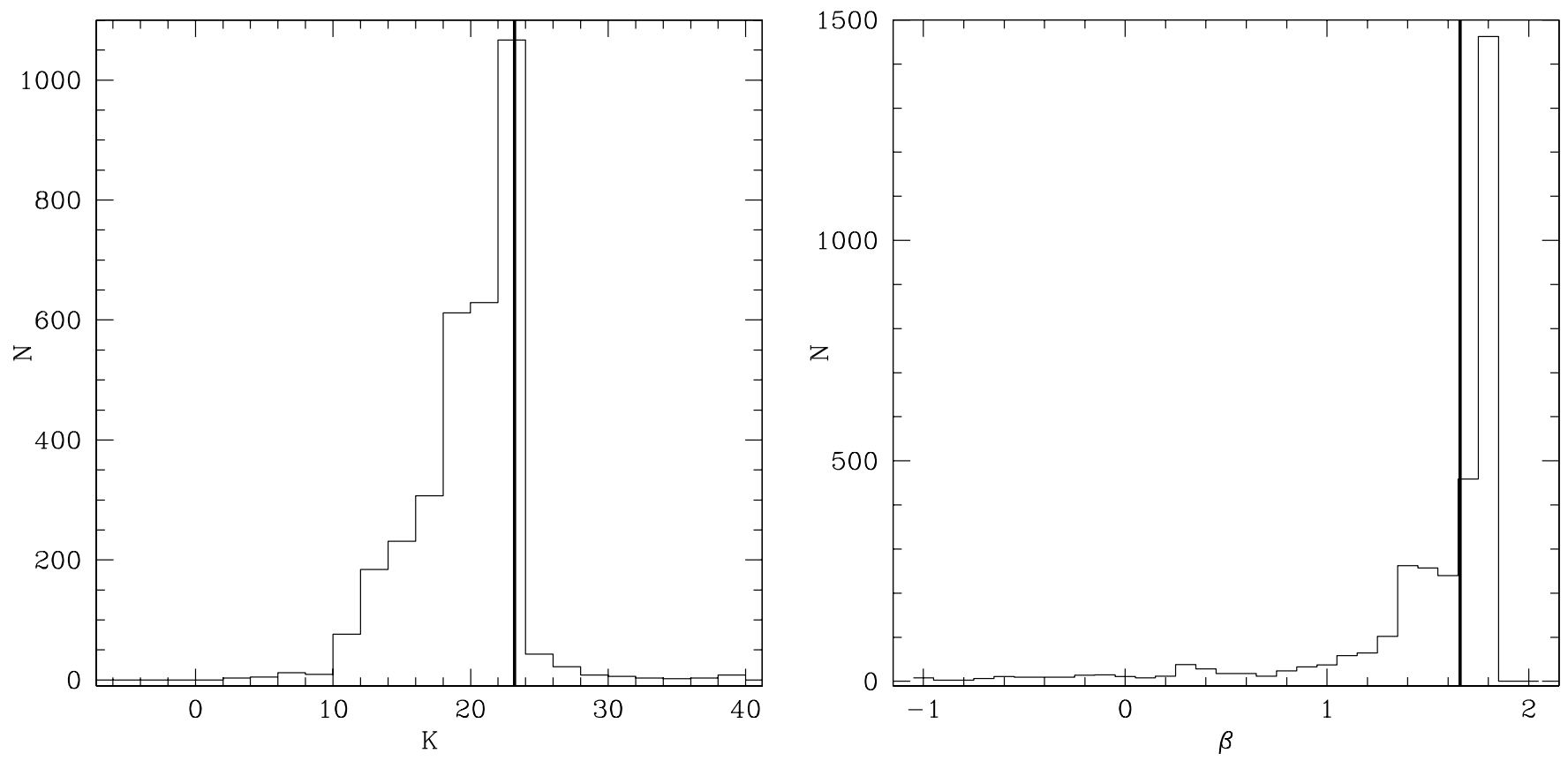

Figure 10. Figures show the range of $K$ and $\beta$ for the chosen parameters in Table 2. $K$ is given in units of Kelvin. The vertical line marks the values of $K$ and $\beta$ in the Analytic Solution.

temperature distribution most accurately with the Fit Solution. There might be some error in the calculation of $K$ and $\beta$ for models with large luminosities or small $\alpha$ 's. However, we do not expect very small values of $\alpha$ to be common in future star formation simulations based on observations of similar regions (Mueller et al. 2002; Shirley et al. 2002; Young et al. 2003).

\section{GAS TEMPERATURE CALCULATION}

After the dust temperature as a function of distance from luminosity sources is derived for positions near stars in a cluster using the look-up table and Equation (18), the gas temperature can be calculated assuming gas energetics balance. We calculate the gas temperature using a gas-dust energetics code which includes energy transfer between gas and dust via collisions, heating by cosmic rays, and molecular cooling (see Doty \& Neufeld 1997 and the appendix in Young et al. 2004 for a more detailed description). We assume that the dust-to-gas mass ratio is $\eta_{\mathrm{dg}}=4.86 \times 10^{-3}$ (Hollenbach \& McKee 1989) and the grain cross section per baryon is $6.09 \times 10^{-22} \mathrm{~cm}^{2}$ (Young et al. 2004). The cosmic ray ionization rate is $3.00 \times 10^{-17} \mathrm{~s}^{-1}$ (van der Tak \& van Dishoeck 2000) and the energy deposited per cosmic ray ionization is $2.00 \times 10^{1} \mathrm{eV}$ (Goldsmith 2001) in 

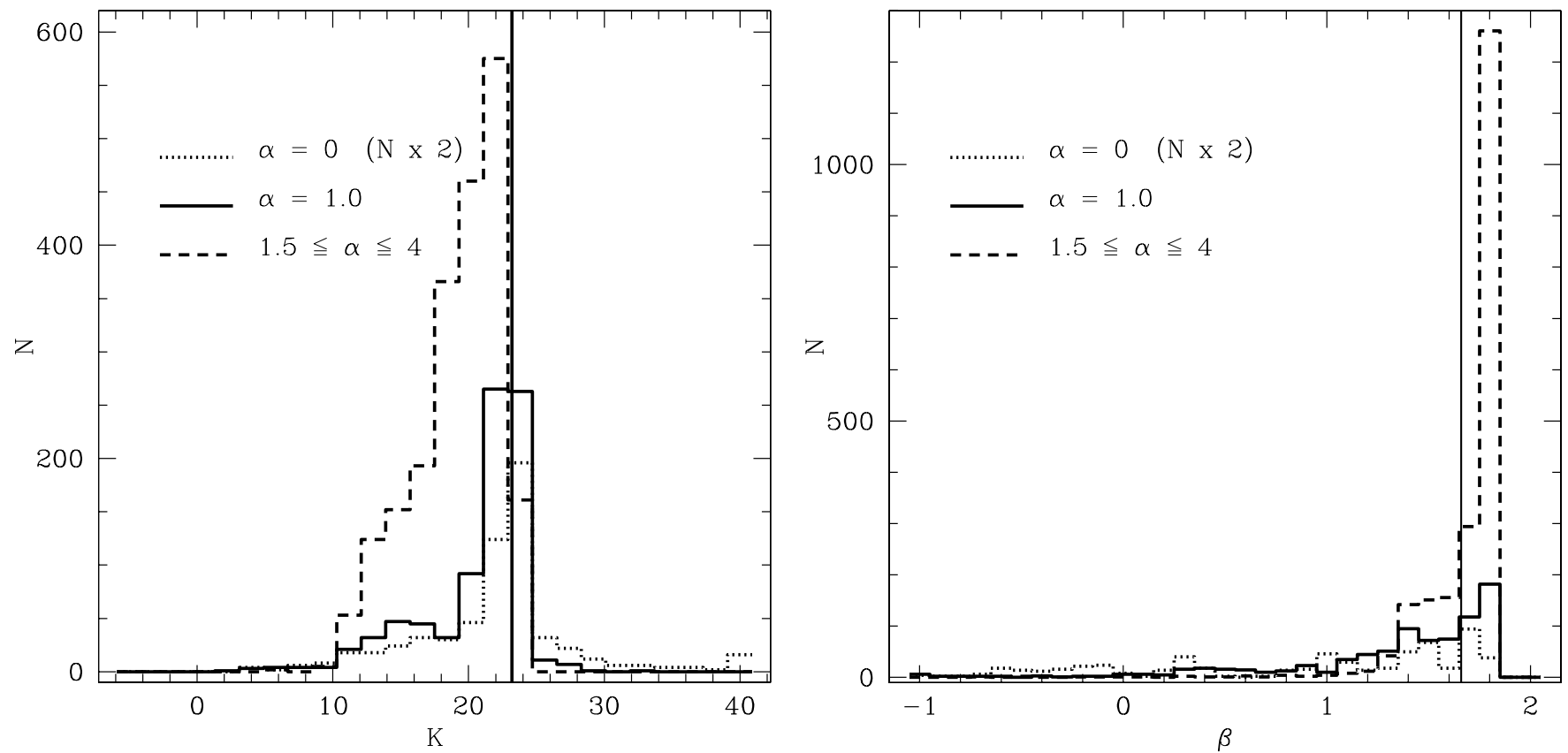

Figure 11. Range of $K$ and $\beta$ for the chosen parameters in Table 2 as a function of $\alpha . K$ is given in units of Kelvin. The vertical line marks the values of $K$ and $\beta$ in the Analytic Solution. Histograms for $\alpha=0$ have been multiplied by 2 for clarity.

our models. We take the fractional abundance of $\mathrm{CO}$ relative to $\mathrm{H}_{2}$ to be $X(\mathrm{CO})=1.0 \times 10^{-4}$ from Figure 8 (a) of Lee et al. (2004).

The model-dependent input parameters are $T_{d}$, local density, column density, and local velocity dispersion $(b) . T_{d}$ is calculated with the procedure described in Section 2.2. Local density and column density can be derived from our input density profile. The column density is calculated radially from the point of interest to the edge of the system. The edge of the system is defined as either the point at which the density is lowest or some fiducial value (as discussed later in Section 4). The velocity-spread parameter, $b$, is defined for a Maxwellian velocity distribution as $b=(2 k T / m)^{1 / 2}$ (Spitzer 1998) and is assumed to be $1 \mathrm{~km} \mathrm{~s}^{-1}$ throughout this paper. The stellar radiation temperature is not a direct input parameter; it is only used to calculate the dust temperature. For the rest of the paper we use the dust models calculated with $T_{*}=5000 \mathrm{~K}$ to determine the dust and gas temperature fields.

We assume that the cluster (defined as a group of small cores which can each be modeled using a set of the parameters defined in Table 2) that we study is deeply embedded within a larger molecular cloud. Therefore, there is no interstellar radiation field impinging on the outer bounds of the cluster and the photoelectric effect on polycyclic aromatic hydrocarbons (PAHs) is not present. Although we have chosen $T_{*}=5000 \mathrm{~K}$ and there is little UV radiation, it is also possible to use a higher stellar temperature, i.e., $T_{*}=10,000 \mathrm{~K}$, which will lead to photoelectric heating of the gas near the inner edge of the envelope surrounding each source. If this higher stellar temperature is used, then our gas temperature calculation is no longer accurate for a subset of our models with low $\tau_{100 \mu \mathrm{m}}$, low $\alpha$, and low $n_{o}$. Also, even at low stellar temperatures, the UV flux from the star may still compete with the dust in heating the gas very close to the star before all the UV has been absorbed by dust. Therefore, we do not claim to correctly calculate the gas temperature within a radius of $\sim 100 \mathrm{AU}$ of each star. In the rest of the paper, we may show the gas temperature for regions closer than $100 \mathrm{AU}$ to a star, however, our calculations do not include photoelectric heating and as such, the actual gas temperature may be higher in this region.

Figure 14 shows the variation of dust and gas temperature fields with distance from a stellar heating source for two values of $X(\mathrm{CO})$. Close to the source, the dust and gas temperature fields are coupled due to collisional interactions of the dust with the gas. As the density decreases, collisions between the dust and gas become less frequent and the gas is able to cool via molecular (mainly $\mathrm{CO}$ ) rotational transitions. Then as the density continues to drop and there is less $\mathrm{CO}$ to cool the gas, cosmic ray heating becomes the dominant heating source and the gas temperature increases. For a gas with less $\mathrm{CO}(X(\mathrm{CO})=$ $\left.5 \times 10^{-5}\right)$, the cooling is not as efficient and the temperature is larger. As various other parameters change in our models, different heating and cooling terms dominate and the minimum and maximum temperatures vary. We discuss this in more detail in the following section (Section 4).

As seen in Figure 14, the gas temperature falls below $10 \mathrm{~K}$ when gas collisions are the dominant cooling method. Our gas cooling rate calculations are based on the work of Neufeld \& Kaufman (1993) and Neufeld et al. (1995) which only extend down to $10 \mathrm{~K}$ because the $\mathrm{H}_{2}-\mathrm{CO}$ collisional rates were not defined below $10 \mathrm{~K}$ at the time of their work. We expect the cooling rate $(\Lambda)$ to drop drastically as the temperature approaches zero and we have attempted to adjust our cooling rate calculation to account for this. We modify our method of calculating the rate of change of the cooling rate with temperature, $\mathrm{d}(\log \Lambda) /$ $\mathrm{d}(\log T)$, by using the Large Velocity Gradient (LVG) model. We extrapolate the CO rate coefficients from Flower \& Launay (1985) from $10 \mathrm{~K}$ to $5 \mathrm{~K}$. Then we calculate $\mathrm{d}(\log \Lambda) /$ $\mathrm{d}(\log T)$ between $10 \mathrm{~K}$ and $5 \mathrm{~K}$ using the LVG model. We apply the new values of $\mathrm{d}(\log \Lambda) / \mathrm{d}(\log T)$ between 10 $\mathrm{K}$ and $5 \mathrm{~K}$ to improve our gas energetics model at low temperatures. 


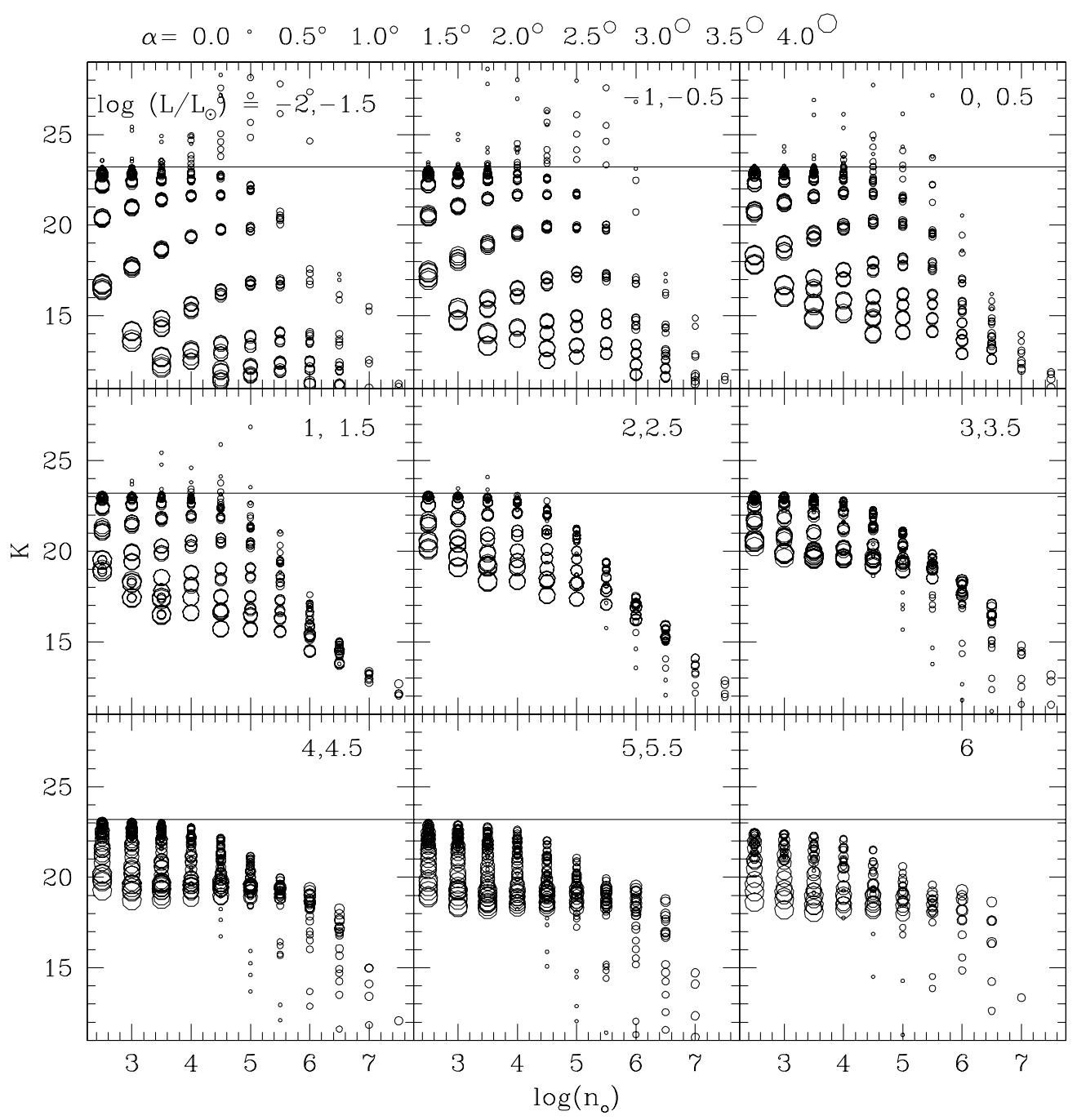

Figure 12. Horizontal line marks the value of $K$ in the Analytic Solution. Individual models are plotted as circles of various sizes. The size of the circle indicates the value of the $\alpha$ parameter as noted in the top of the plot. The nine separate plots each show $K$ as a function of $n_{o}\left(\mathrm{~cm}^{-3}\right)$ for nine different luminosity regimes. In the top-left box, $\log L / L_{\odot}$ is -2 or -1.5 , as indicated at the top-right corner in the box. The bottom-right box shows models with the highest luminosities. $K$ is given in units of Kelvin.

\section{GAS AND DUST TEMPERATURE WITH MULTIPLE SOURCES}

The goal of our paper is to calculate the dust and gas temperature fields in a field of stars that are forming. In such an environment, individual stars are surrounded by an envelope of dust and gas which feeds their growth. In this section we describe the dust and gas temperature fields in a field of two (Section 4.1) and three (Section 4.2) sources to illustrate our method. With our method, we can solve for the gas and dust temperature in a field of many sources.

\subsection{Two Sources}

In order to calculate the gas temperature between two sources, we must first calculate the dust temperature. We do this using the method described in Section 2.2. Once we have determined the dust temperature, we can use our energetics algorithm to calculate the gas temperature. Around each source we place a density profile. In order for this to be realistic, we choose a density, $n_{\mathrm{eq}}$ at which we have the two density profiles meet. The value of $n_{\text {eq }}$ sets the distance between the sources, i.e., smaller values of $n_{\text {eq }}$ place the sources farther apart.
Figure 15 shows the dust and gas temperature profile for increasing values of $n_{\mathrm{eq}}$, i.e., smaller separations. An interesting feature of this plot is that if we only look at the region between the two sources, we find a large variation in the gas temperature. This is due to the higher densities sampled as the sources move closer together. The top panel shows sources that are far apart and we see that the maximum gas temperature between the two sources is $\sim 20 \mathrm{~K}$ and the minimum gas temperature is $\sim 7 \mathrm{~K}$. Cosmic ray heating, though relatively weak, can warm the material sufficiently far from luminous sources. As the sources move closer together, cosmic rays become less important until the temperature between the sources ceases to increase, whereas dust heating becomes more important and the gas is not able to cool efficiently and the minimum temperature between the sources rises.

\subsection{Three Sources}

In this section, we calculate the gas and dust temperature distribution around three sources. The three sources were placed on three of the corners of a square with sides of length $1000 \mathrm{AU}$. The least luminous source was placed on the corner between the 


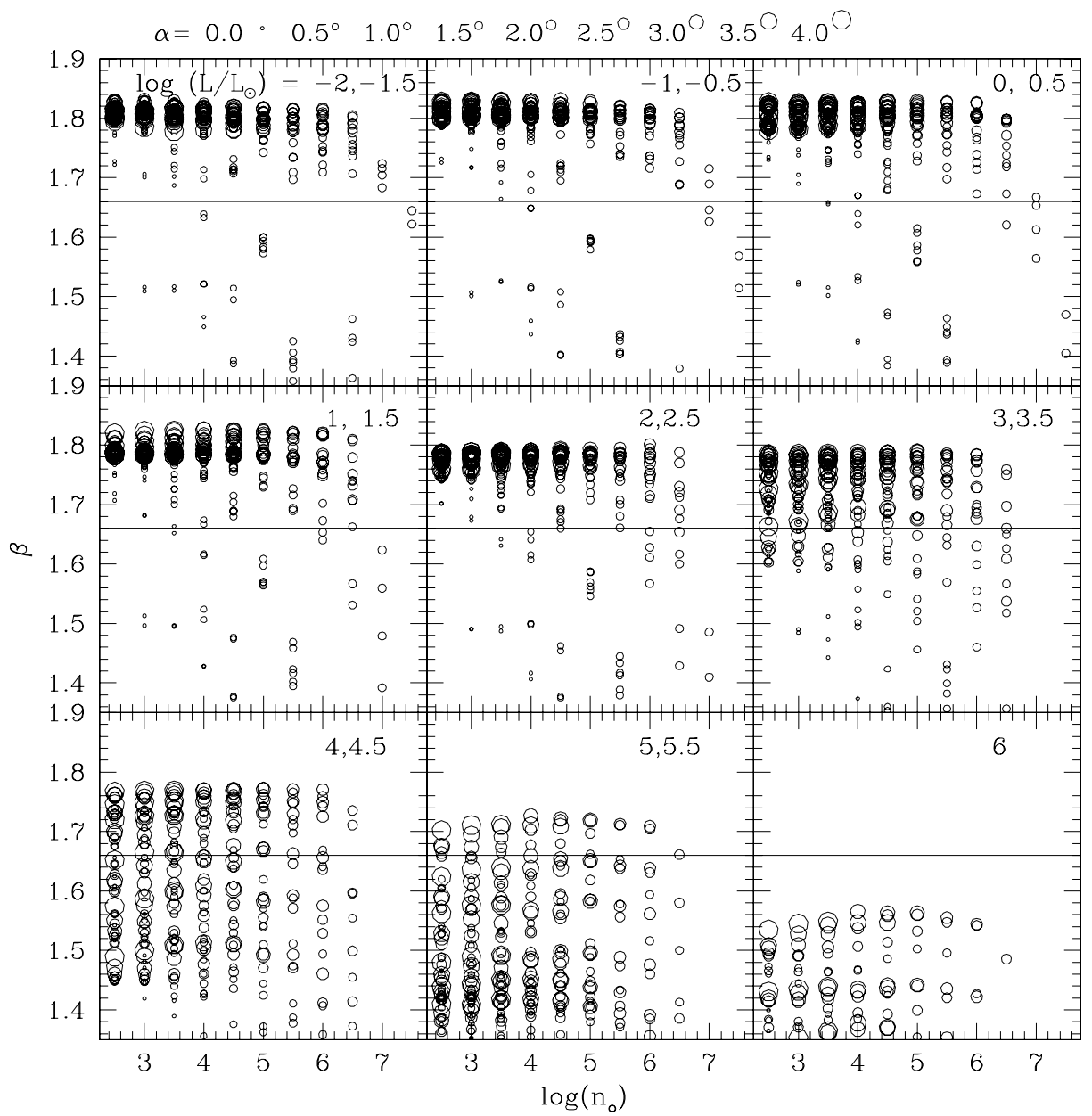

Figure 13. Horizontal line marks the value of $\beta$ in the Analytic Solution. Same plot details as Figure 12 except $\beta$ is plotted rather than $K$.

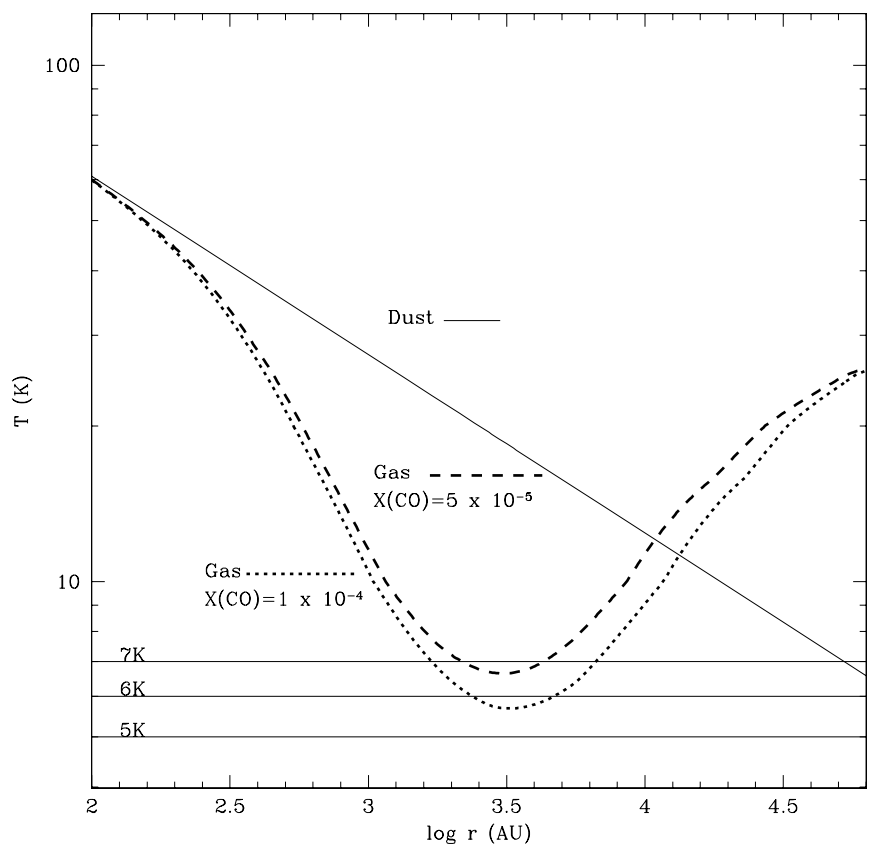

Figure 14. Gas temperature distribution and comparison of difference in $\mathrm{CO}$ abundance. Solid line shows Fit Solution to dust temperature. The model parameters are $\log \left(L / L_{\odot}\right)=1, \log \left(r_{\text {out }} / 1 \mathrm{pc}\right)=-0.5, \log \left(n_{o} / \mathrm{cm}^{-3}\right)=4.5$, and $\alpha=2.5$. Dotted and dashed lines show gas temperature for $X(\mathrm{CO})=$ $1 \times 10^{-4}$ and $X(\mathrm{CO})=5 \times 10^{-5}$, respectively.
Table 3

Source Parameters

\begin{tabular}{lcccccccc}
\hline \hline Source Luminosity $\left(L_{\odot}\right)$ & $n_{o}\left(\mathrm{~cm}^{-3}\right)$ & $\alpha$ & $r_{\text {out }}(\mathrm{pc})$ & $K(\mathrm{~K})$ & $\beta$ & $x(\mathrm{AU})$ & $y(\mathrm{AU})$ \\
\hline 1 & 1 & $10^{3}$ & 2 & 0.1 & 22.9 & 1.78 & 500 & 1500 \\
2 & 100 & $10^{5}$ & 2 & 0.1 & 19.6 & 1.76 & 1500 & 1500 \\
3 & 10 & $10^{4}$ & 2 & 0.1 & 21.9 & 1.78 & 500 & 500 \\
\hline
\end{tabular}

other two sources. For simplicity, we only calculate the dust and gas temperature fields in the plane occupied by all three sources. The same method of calculating the temperature in the plane can be used to calculate the temperature at any point above or below the sources.

The positions (on a $2000 \mathrm{AU} \times 2000 \mathrm{AU}$ grid), luminosities, and density profiles of each source are given in Table 3 . The density profiles of the sources are not consistent with each other; each point in the grid has three density values associated with it, one for each of the three sources. We choose to calculate the density in this manner because this is how we expect our dust/ gas temperature algorithm to be used in a large-scale simulation. For each source in the simulation, the spherical density profile surrounding that source will be calculated and used to find the dust temperature and, using the method described in this section, the gas temperature.

We calculate the dust temperature using the method described in Section 2.2 and show the results in Figure 16. In order to 

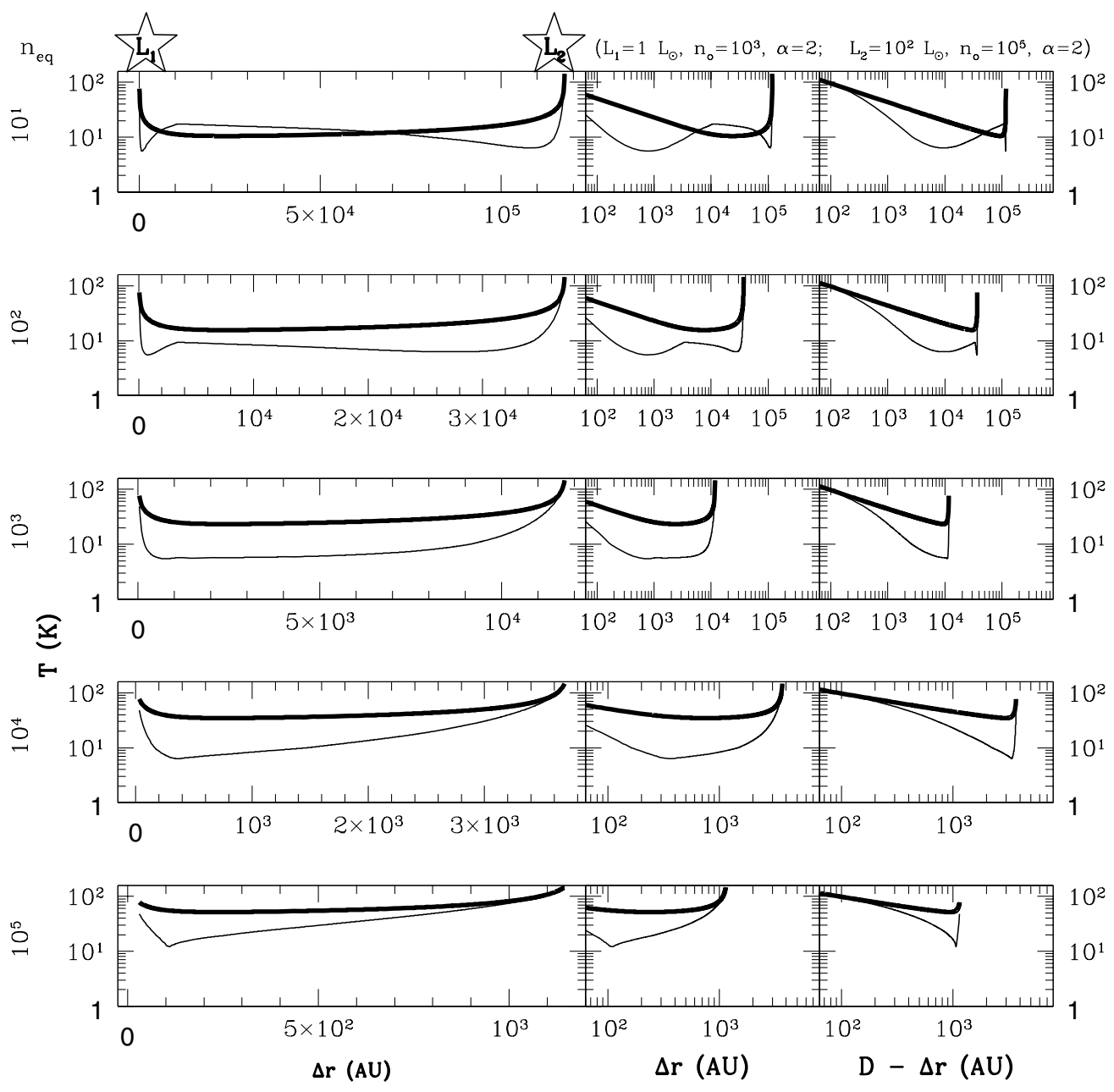

Figure 15. Gas and dust temperature distribution between two sources. Plots show dust (thick line) and gas (thin line) temperature as a function of distance between two stellar sources. From top to bottom, distance between sources is decreasing, such that the gas density surrounding the two sources agrees with the value, $n_{\text {eq }}$ quoted on the left. The source on the left (1) has the parameters $L=1 L_{\odot}, n_{o}=10^{3} \mathrm{~cm}^{-3}$, and $\alpha=2$. The right source (2) has $L=10^{2} L_{\odot}, n_{o}=10^{5} \mathrm{~cm}-3$, and $\alpha=2$. The three horizontal plots for the different values of $n_{\text {eq }}$ show the same data from different perspectives. On the longer plot on the left we use a linear scale from the perspective of source 1 . The two smaller plots on the right use a logarithmic scale, from the perspective of source 1 (middle) and source 2 (right). This is done to show structure close to the individual sources.

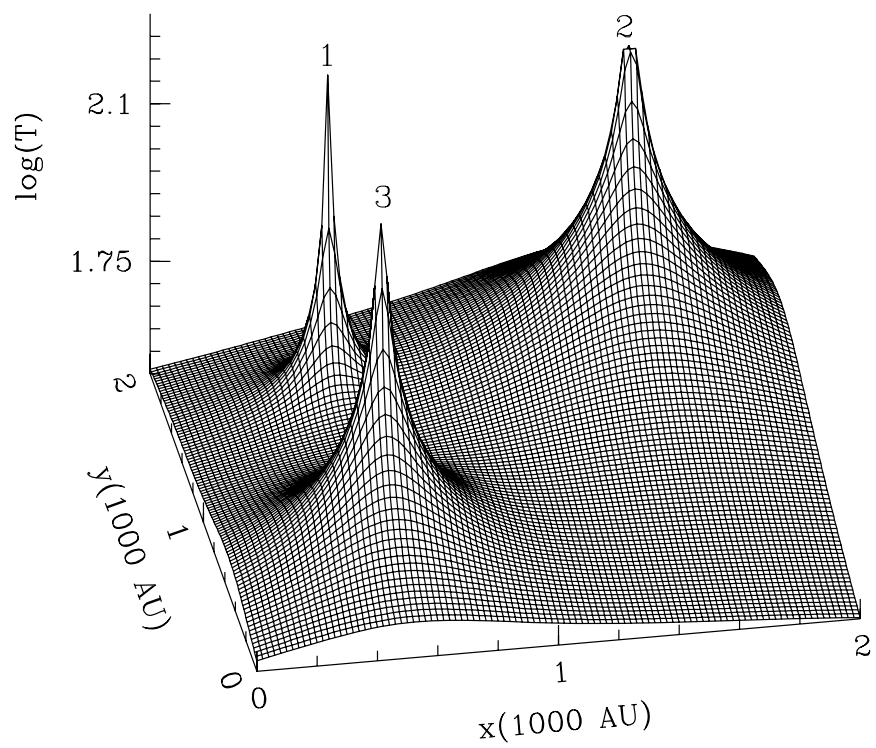

Figure 16. Surface plots of dust temperature (K). Sources are labeled according to the parameters listed in Table 3. determine the gas temperature, we first calculate the density $(\rho)$ and column density $\left(N_{\mathrm{col}}\right)$ at each point on the grid due to all three sources; every point on the grid has three possible values of $\rho$ and $N_{\text {col }}$. The values of density are calculated from the three different input density profiles. $N_{\text {col }}$ is calculated by integrating the three different density profiles from the point of interest radially away from the source to the edge (the edge is defined in the next paragraph). Then, at each point we choose the source which gives the highest value of $N_{\mathrm{col}}$ at that point and use that source to calculate $N_{\mathrm{col}}, \rho$, and the gas temperature for that specific point on the grid. We choose the highest column density because that value is most likely to represent the column density at that point on the grid. The lower values of column density are likely to come from calculating the column density from the tail of the density profile of another, more distant source. Figure 17 demonstrates visually the method that we use to select density and column density and then calculate the gas temperature. This figure shows that near source $1(x=500)$, the density calculation is dominated by the profile of source 2 , until the region very close to source 1 is considered.

As mentioned before, we calculate $N_{\text {col }}$ by integrating from the point of interest to the "edge" in the direction radially 

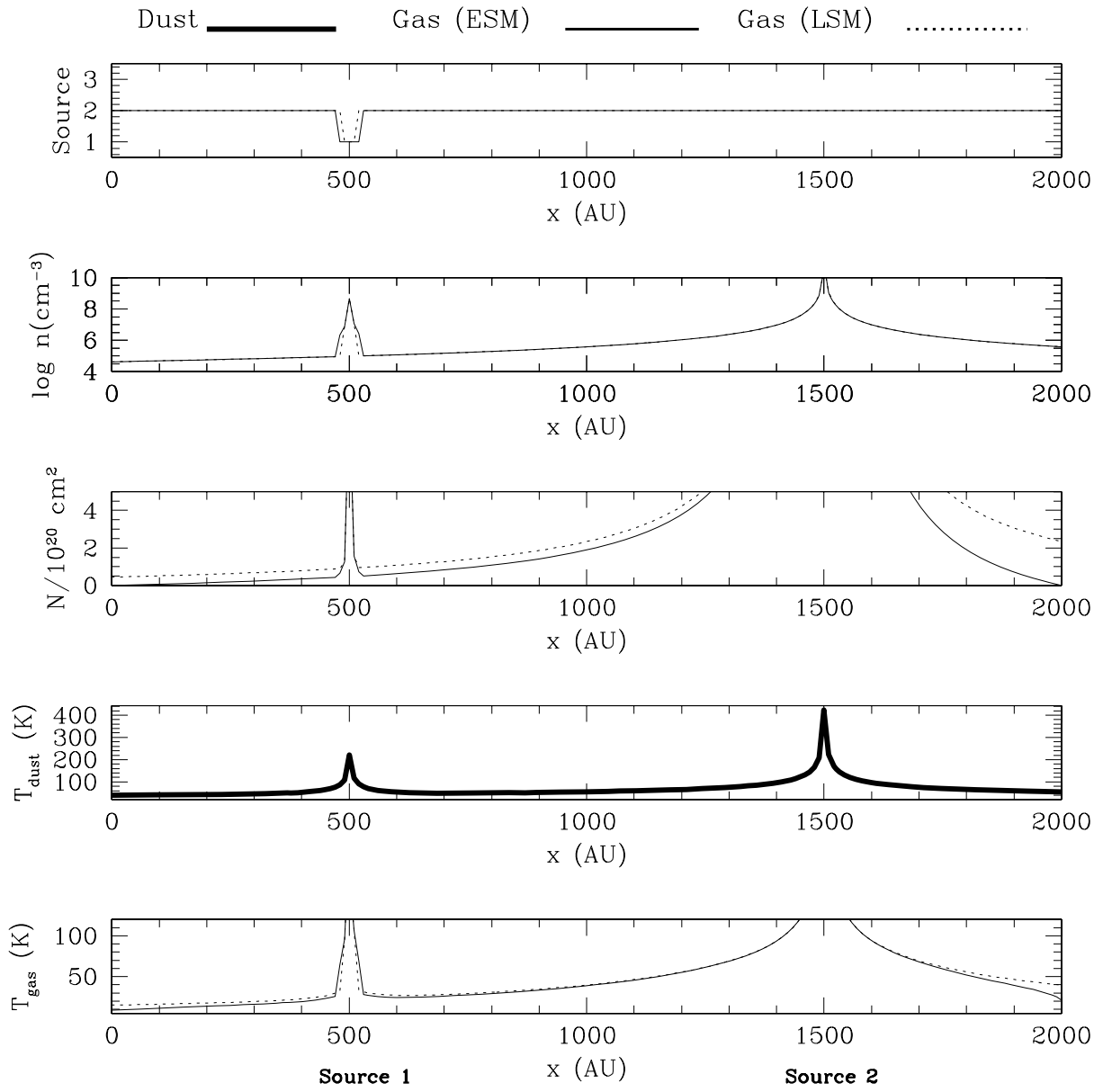

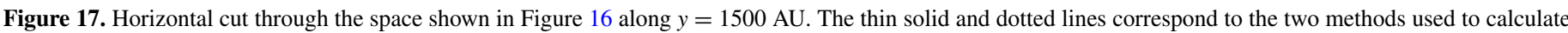

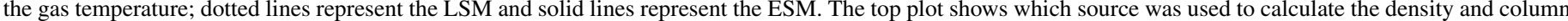

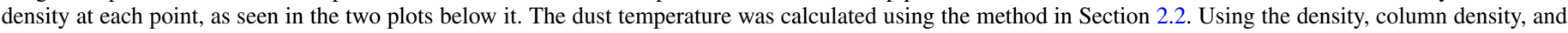
dust temperature, the gas temperature is calculated and plotted in the bottom plot.

away from the source. We tested two methods of defining the "edge." Our first method, the "Length of Square Method (LSM)," integrates from the point of interest to $2000 \mathrm{AU}$ from each source (Figures 18 and 19). The value of $2000 \mathrm{AU}$ was chosen arbitrarily to equal the length of the side of the square in which we placed our sources. Our second method, the "Edge of Square Method (ESM)," integrates from the point of interest to the edge of the $2000 \mathrm{AU} \times 2000 \mathrm{AU}$ grid (Figures 18 and $20)$. Using this method, the integration length depends on the direction of integration.

In both cases, we find that the gas and dust temperature become equal to one another in regions of high density close to a luminosity source as seen in Figure 18 at $T=100 \mathrm{~K}$. In these high density regions, the dust and gas temperature fields are coupled through collisions. As the density decreases, the gas temperature drops rapidly, compared to the dust temperature, due to the ability of the gas to cool through molecular transitions. Another interesting feature of these plots is the visibility of source 1, the dimmest source in the region, even though it is close to source 2, the brightest source in the region.

Two differences in the gas temperature between the two methods of calculating $N_{\text {col }}$ are evident in Figure 18. The first is the square shape of the contours near the edges when using the ESM. This is an artifact of a square grid. The second difference is the increasing discrepancy between the two methods at large distances from the sources (or at locations where the density is low). This difference is due to the different methods of

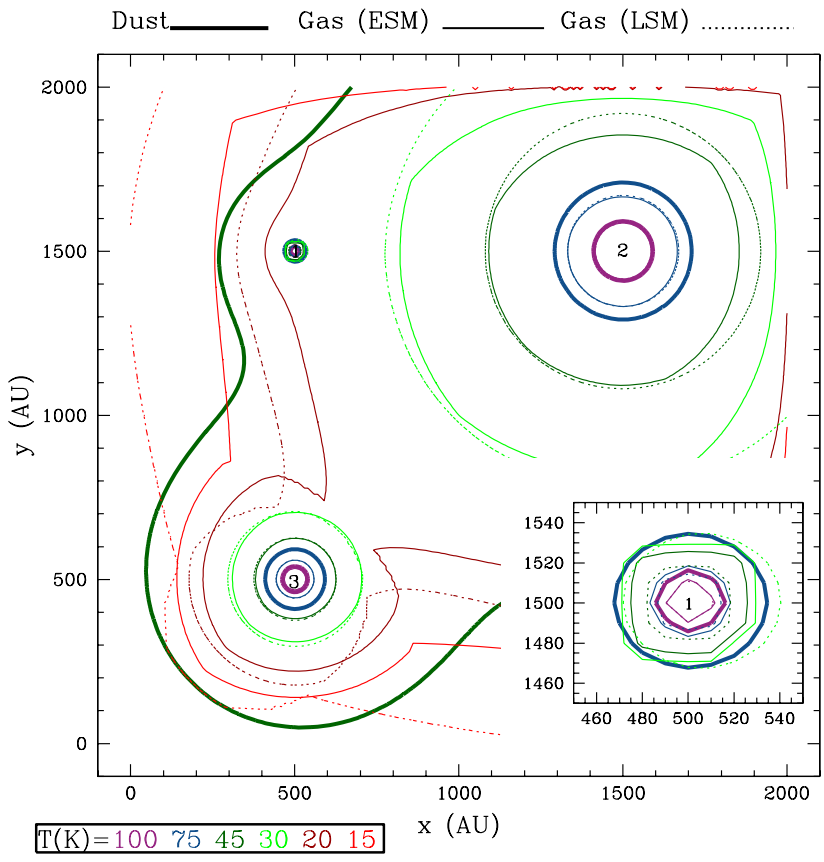

Figure 18. Contour plot of dust and gas temperature fields using the LSM and the ESM. Sources are labeled according to the parameters listed in Table 3. Inset box shows detail of region near source 1. As discussed in Section 3, the gas temperature values within $100 \mathrm{AU}$ of each source may not be correct.

(A color version of this figure is available in the online journal.) 


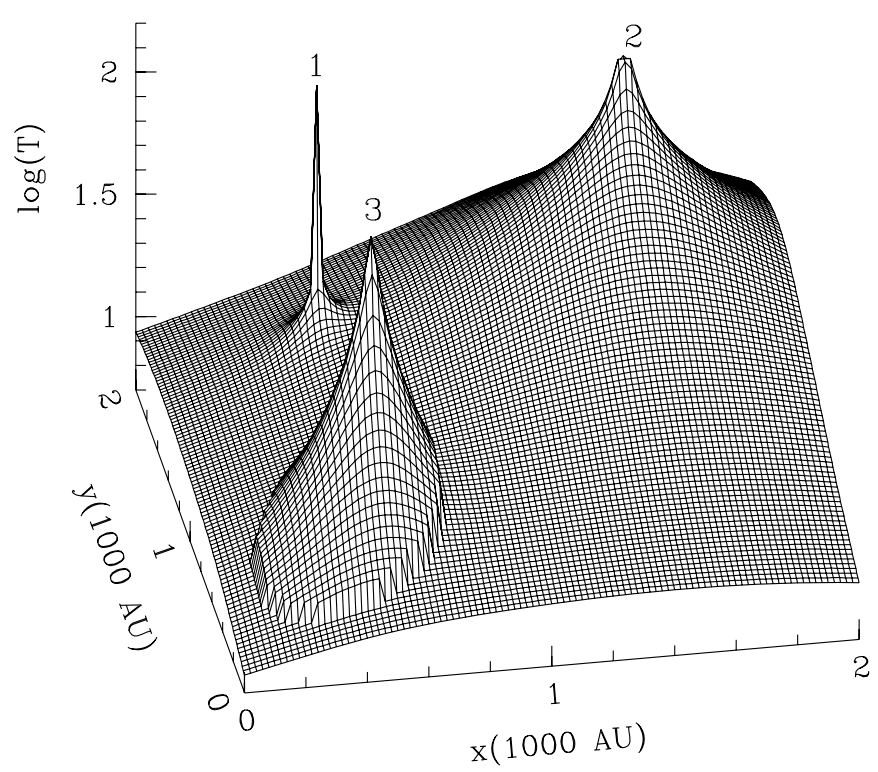

Figure 19. Surface plots of gas temperature (K) using the LSM. The gas temperature values within $100 \mathrm{AU}$ of each source may not be correct as discussed in Section 3.

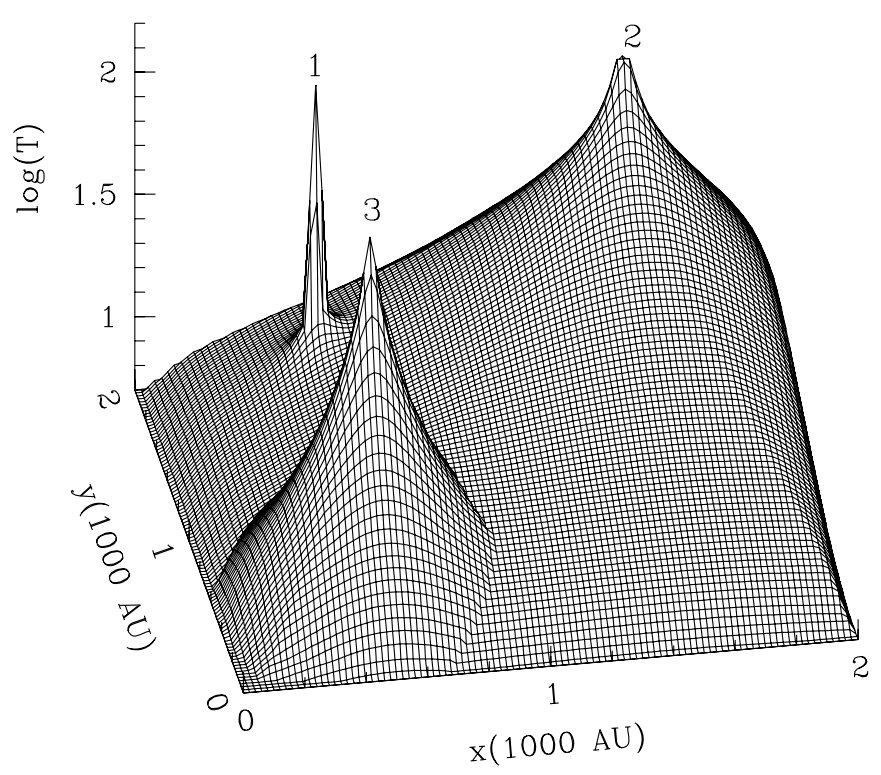

Figure 20. Similar to Figure 19 except for the ESM.

calculating the column density, which depends on the local density. At positions near the edge of box and also far away from a source, the calculation of the column density will be the most different between the two methods. This is because the LSM will integrate the density to $2000 \mathrm{AU}$ in order to determine the column density, but the ESM will only integrate to the edge of the square which can be as little as $10 \mathrm{AU}$. This leads to gas temperatures that are most different near the edge, i.e., the gas temperature calculated with the LSM is greater than the gas temperature determined using ESM. Throughout the rest of this paper, we use the LSM to calculate the gas temperature. However, it is important to remember that both of these methods only provide an approximation of the gas temperature and are used because they are quick and easy to calculate.

In Figure 21, we compare the gas and dust temperatures far from the sources when we remove two of the three sources, leaving only source 2 . The similarity of the dust and gas

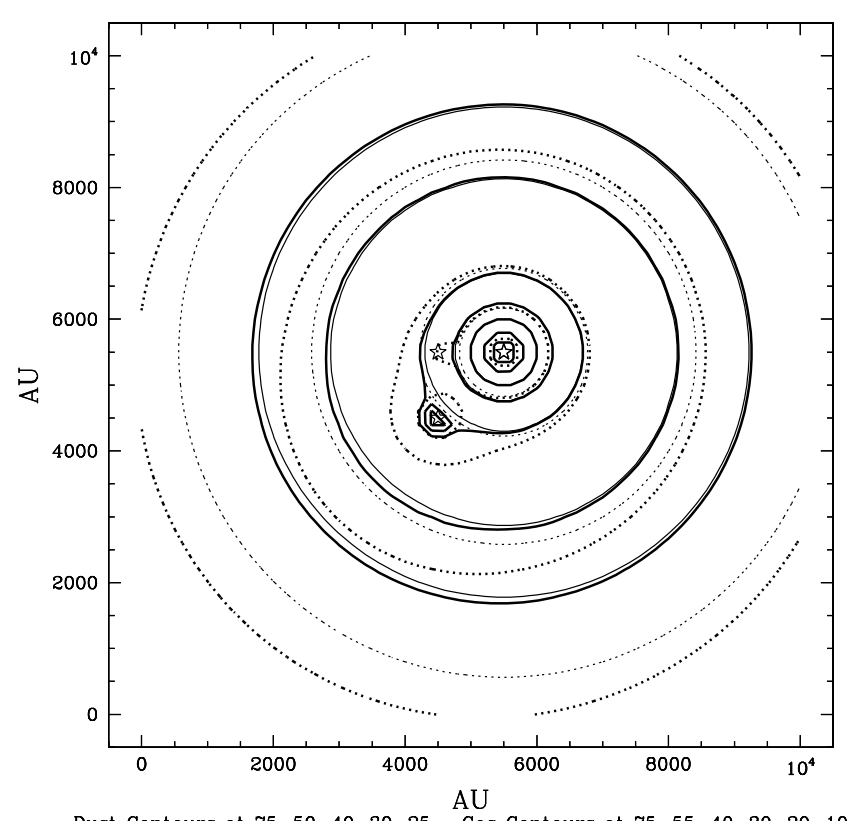

.... Dust Contours at 75, 50, 40,30, 25 _-Gas Contours at 75, 55, 40,30, 20, 10, 8

Figure 21. Contour plots of dust and gas temperature fields with three sources (thick lines) and one source (thin lines). Contours of $T_{d}(\mathrm{~K})$ are shown as dashed lines, while contours of gas temperature $(\mathrm{K})$ are shown as solid lines.

temperature contours illustrates the difficulty in determining the number of sources responsible for heating the gas and dust and highlights the need for adequate spatial resolution in observations.

In Figure 22, we have zoomed out of the region of interest. Note that the dust temperature steadily decreases as the distance from the central three sources increases. Yet, the gas temperature slowly begins to rise, because of the decreased effectiveness of $\mathrm{CO}$ cooling at low densities.

\section{CONCLUSION}

We have presented a method for calculating the dust and gas temperature fields between stellar sources. The analytic method that we investigated for calculating the dust temperature was not accurate enough. Instead, our chosen method of calculating the dust temperature uses a simple radiative transfer code which we use to create a look-up table. Once we have derived the dust temperature, we are able to calculate the gas temperature by balancing various energy processes. We include dust-gas collisional heating, molecular cooling, and cosmic-ray heating. When we have balanced the energies, we are able to derive the gas temperature.

We plan to use the method described in this paper to model a region of clustered star formation with the threedimensional hydrodynamics code discussed in Martel et al. (2006). Klessen et al. (1998) simulate a low density region and assume that the gas is isothermal. At higher densities, Krumholz et al. (2007) assume that the dust and gas temperature fields are coupled. In order to model regions of intermediate densities, neither approach is valid. In Figure 23, we show the temperature difference between the gas and dust temperatures as a function of the density and dust temperature for the sources discussed in Section 4.2, excluding regions within $100 \mathrm{AU}$. The branches extending to the right in this figure show the behavior of the temperature and density close to the two brightest sources in the region, Sources 2 and 3. We 

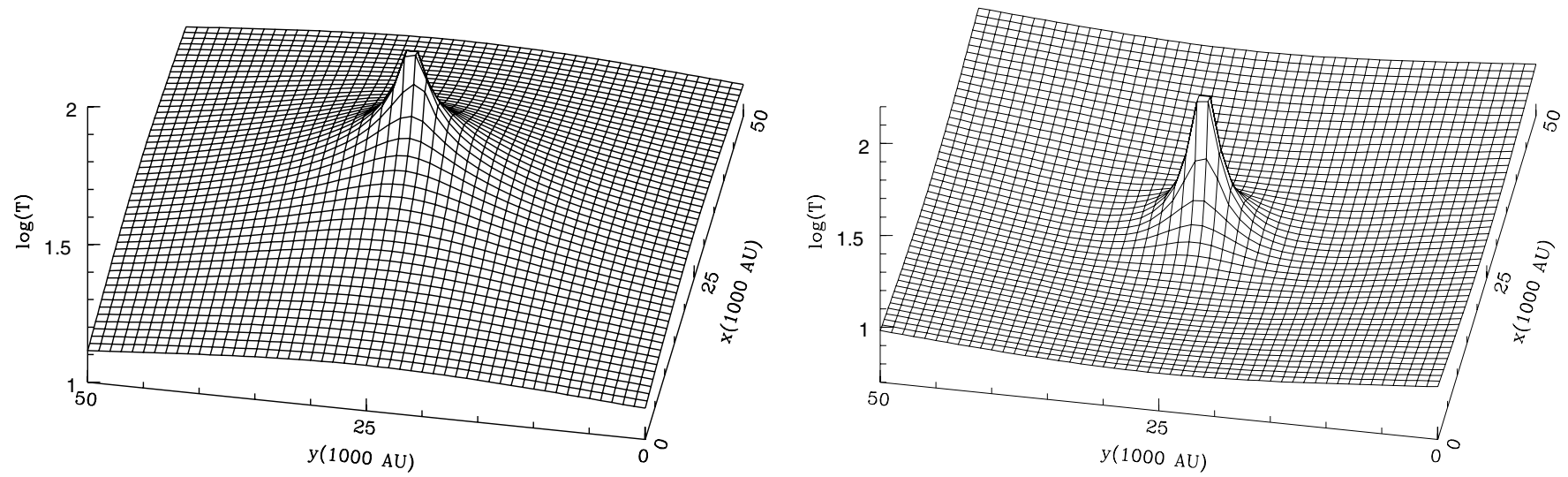

Figure 22. Surface plots of dust and gas temperature fields. The left plot shows dust temperature (K). The right plot shows gas temperature (K). Area sampled is $50,000 \times 50,000 \mathrm{AU}$. The LSM is used here.
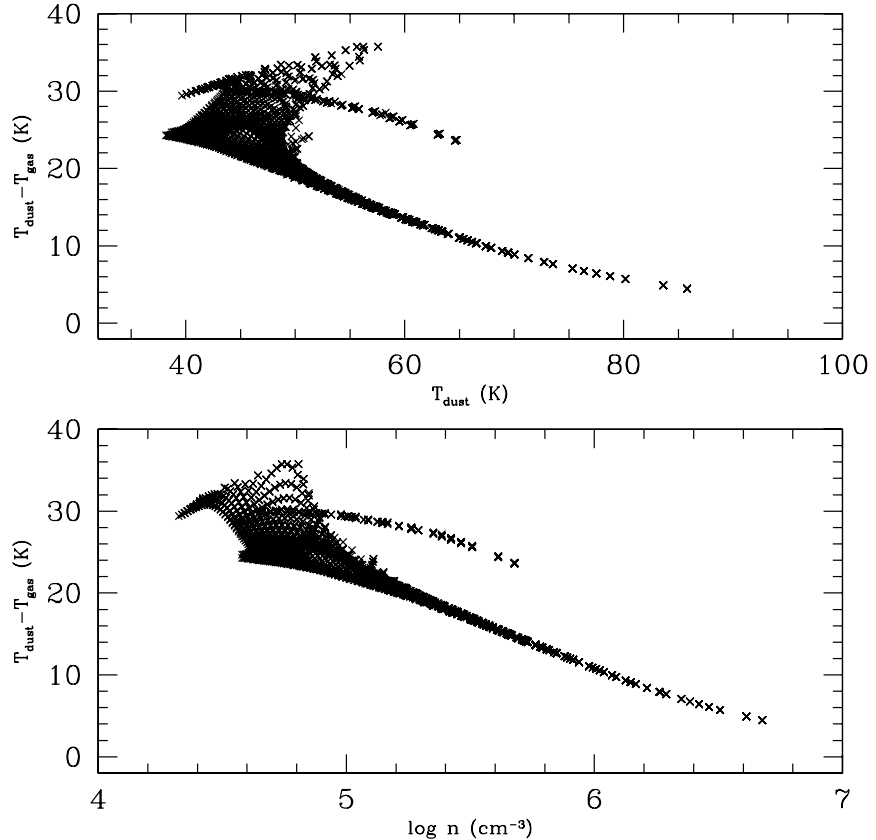

Figure 23. Difference in dust and gas temperature fields plotted for the region shown in Figures 16-19, excluding the volume within 100 AU of each source. The top plot shows temperature difference as a function of dust temperature. The bottom plot shows temperature difference as a function of density.

find that the dust and gas temperature fields are nearly equal at very high densities $\left(n>10^{7} \mathrm{~cm}^{-3}\right)$. However, at $n \sim$ $10^{5} \mathrm{~cm}^{-3}$ the difference between the dust and gas temperature fields is $>20 \mathrm{~K}$ with a considerable spread. (The previous two statements are only valid for the gas and dust temperatures around low luminosity protostars since UV heating will become important for the gas around high luminosity protostars.) The spread shows that the gas temperature is not only determined by the local density, but also by other factors such as nearby stellar sources and the surrounding density field. Therefore, in the intermediate density regime we show in the figure, a formulaic method of calculating the dust and gas temperature fields is inappropriate. Our method of calculating the gas and dust temperature distribution in a field of young stars will enable us and others to more accurately model clustered star formation in future simulations.

A.U. would like to thank the NASA GSRP for providing support and Chad Young and Jeong-Eun Lee for help with DUSTY and the gas energetics code. N.E. would like to thank the NSF for grants AST-0307250 and AST-0607793. This work was partially supported by a grant from The Research Corporation (SDD).

\section{REFERENCES}

Bate, M. R., Bonnell, I. A., \& Bromm, V. 2003, MNRAS, 339, 577

Doty, S. D., \& Neufeld, D. A. 1997, ApJ, 489, 122

Flower, D. R., \& Launay, J. M. 1985, MNRAS, 214, 271

Goldsmith, P. F. 2001, ApJ, 557, 736

Hildebrand, R. H. 1983, QJRAS, 24, 267

Hollenbach, D., \& McKee, C. F. 1989, ApJ, 342, 306

Klessen, R. S., Burkert, A., \& Bate, M. R. 1998, ApJ, 501, L205

Krumholz, M. R., Klein, R. I., \& McKee, C. F. 2007, ApJ, 656, 959

Lada, C. J., \& Lada, E. A. 2003, ARA\&A, 41, 57

Lee, J.-E., Bergin, E. A., \& Evans, N. J., II. 2004, ApJ, 617, 360

Makinen, P., Harvey, P. M., Wilking, B. A., \& Evans, N. J., II. 1985, ApJ, 299, 341

Martel, H., Evans, N. J. II, \& Shapiro, P. R. 2006, ApJS, 163, 122

Mueller, K. E., Shirley, Y. L., Evans, N. J. II, \& Jacobson, H. R. 2002, ApJS, 143,469

Nenkova, M., Ivezić, Ž., \& Elitzur, M. 2000, in ASP Conf. Ser. 196, Thermal Emission Spectroscopy and Analysis of Dust, Disks, and Regoliths, ed. M. L. Sitko, A. L. Sprague, \& D. K. Lynch (San Francisco, CA: ASP), 77

Neufeld, D. A., \& Kaufman, M. J. 1993, ApJ, 418, 263

Neufeld, D. A., Lepp, S., \& Melnick, G. J. 1995, ApJS, 100, 132

Ossenkopf, V., \& Henning, T. 1994, A\&A, 291, 943

Press, W. H., Teukolsky, S. A., Vetterling, W. T., \& Flannery, B. P. 1992, Numerical Recipes in FORTRAN 77. The Art of Scientific Computing (2nd ed.; Cambridge: Cambridge Univ. Press)

Pollack, J. B., Hollenbach, D., Beckwith, S., Simonelli, D. P., Roush, T., \& Fong, W. 1994, ApJ, 421, 615

Shirley, Y. L., Evans, N. J. II, \& Rawlings, J. M. C. 2002, ApJ, 575, 337

Spitzer, L. 1998, Physical Processes in the Interstellar Medium (New York: Wiley-VCH)

van der Tak, F. F. S., \& van Dishoeck, E. F. 2000, A\&A, 358, L79

van der Tak, F. F. S., van Dishoeck, E. F., Evans, N. J. II, Bakker, E. J., \& Blake, G. A. 1999, ApJ, 522, 991

Young, C. H., \& Evans, N. J. 2005, ApJ, 627, 293

Young, K. E., Lee, J.-E., Evans, N. J., Goldsmith, P. F., \& Doty, S. D. 2004, ApJ, 614,252

Young, C. H., Shirley, Y. L., Evans, N. J. II, \& Rawlings, J. M. C. 2003, ApJS, 145,111 